\title{
Employing Unmanned Aerial Vehicles for Improving Handoff using Cooperative Game Theory
}

\author{
S. Goudarzi, Member, IEEE, M. H. Anisi, Senior Member, IEEE, D. Ciuonzo, Senior Member, IEEE, \\ S. A. Soleymani, Member, IEEE, A. Pescapé, Senior Member, IEEE
}

\begin{abstract}
Heterogeneous wireless networks that are used for seamless mobility are expected to face prominent problems in future 5G cellular networks. Due to their proper flexibility and adaptable preparation, remote-controlled Unmanned Aerial Vehicles (UAVs) could assist heterogeneous wireless communication. However, the key challenges of current UAV-assisted communications consist in having appropriate accessibility over wireless networks via mobile devices with an acceptable Quality of Service $(\mathrm{QoS})$ grounded on the users' preferences. To this end, we propose a novel method based on cooperative game theory to select the best UAV during handover process and optimize handover among UAVs by decreasing the $(i)$ end-to-end delay, ( $i$ i) handover latency and ( $i$ ii) signaling overheads. Moreover, the standard design of Software Defined Network (SDN) with Media Independent Handover (MIH) is used as forwarding switches in order to obtain seamless mobility. Numerical results derived from the real data are provided to illustrate the effectiveness of the proposed approach in terms of number of handovers, cost and delay.
\end{abstract}

Index Terms-MIH, game theory, heterogeneous wireless networks, network selection, SDN, UAVs, vertical handover.

\section{INTRODUCTION}

$\mathbf{T}$ HE AIM of future 5G cellular networks is to provide full support for new paradigms such as Internet of Things (IoT). The main goal of $5 \mathrm{G}$ cognitive radio in heterogeneous networks is to sustain seamless connectivity and offer satisfying Quality-of-Service (QoS) by shifting from one network to another via Vertical Handovers (VHO).

Currently, most mobile network operators are expecting an effective mechanism to sustain mobile nodes during VHO setups [1], [2]. In a heterogeneous network (i.e. made of macro base stations, as well as small/pico/femto cells) [3][6], the utilization of dynamic nodes can be considered as an appealing alternative solution to support users' mobility in the case of network traffic. In this respect, an Unmanned Aerial Vehicle (UAV) represents an appealing instance of dynamic node which can be simply managed as a promising solution to satisfy several challenging requirements for the $5 \mathrm{G}$ wireless networks, such as high-speed data transmission and ubiquitous radio coverage [7]. UAVs-assisted networks are usually termed

Sh. Goudarzi is with Centre for Artificial Intelligent (CAIT), Universiti Kebangsaan Malaysia, 43600 Bangi, Malaysia. (e-mail: shidrokh@ukm.edu.my).

M. H. Anisi is with the School of Computer Science and Electronic Engineering, University of Essex, Colchester CO4 3SQ, United Kingdom (email: m.anisi@essex.ac.uk)

D. Ciuonzo and A. Pescap/'e are with the University of Napoli "Federico II”, Italy (e-mail: domenico.ciuonzo@unina.it; pescape@unina.it).

S. A. Soleymani is with the School of Computing, Faculty of Engineering, Universiti Teknologi Malaysia, Johor, Malaysia (e-mail: asseyed4@live.utm.my). as "drone cell networks". Such networks can offer better services than traditional networks because of agility of the UAVs and their dynamic behavior during network operations.

The widespread research studies from academia have used UAVs as various kinds of wireless communication platforms [8] such as aerial mobile base stations (BSs) [9]-[11], mobile relays [12], and flying computing cloudlets [13]. The authors in [9] provided an analytical method for optimizing the altitude of a UAV to offer better coverage for users. The horizontal positions of UAVs are optimized in [14] by fixing the altitude to reduce the number of required UAV BSs for covering a set of ground users [15]-[17]. A UAV-enabled cell employment optimization problem is examined in [18] to exploit the users which can cover in three-dimensional (3D) space. The usage of dynamic nodes has a significant effect on communication systems in solving problems related to high stream data transfers [19]-[21].

Most of the current works in the literature for usage of UAVs in the fifth-generation $(5 \mathrm{G})$ have focused on the concepts related to deployment, capacity, and network coverage [22], [23]. These issues are important, but firstly, a proper target for the efficient management of UAV networks should be identified. Software-Defined Networking (SDN) is a new paradigm where the control of computer and communication networks is completed via programmatic interfaces [24], [25]. Most modern networking companies have utilized SDN as a reliable and efficient mechanism to facilitate the growing demands of network users [25]-[27].

In fact, utilizing UAVs can improve the performance of the existing cellular systems. However, this imposes major challenges with handovers. The most favored characters in the next generation wireless system are the capability to seamlessly move from different access networks over any type of network infrastructure. Therefore, seamless mobility requires VHO. Since the UAVs can be employed as aerial access points, a heterogeneous network requires handover management. The handover among UAVs involves replicating the exact operational state in the incoming UAV containing packets in the buffer, forwarding tables, and data fusion that speeds up the messaging between the UAVs [28]. Data fusion and sharing combining various sources of data available to make a bridge between different types of information technologies. There is a tradeoff between the benefit of aerial stability through handover-related messaging with low transmission power and the long duration to complete the entire handover process [14], [29], [30].

Indeed, UAVs can act as communication hot spots or relays 
along with the heterogeneous networks to solve the problems such as frequent handover, handover failure due to Doppler frequency shift, and low data rate caused by fast fading and multipath loss. Equally important, compared with cellular networks, a single UAV can (a) cover a larger area and (b) provide a higher data rate for a specific user, which is crucial for heterogeneous networks. In these scenarios, the Ground Station (GS) has control over related UAVs and the capability to forward or receive large data traffic. Wireless access technologies need to use UAVs for increasing communication range in the upcoming 5G wireless networks [19], [31]. There have been few studies on the seamless handover in UAV networks to adapt to dynamic topology, intermittent links, and high mobility. There is increasing attention from research community on designing the most efficient routing protocol [32]. However, to fully get the benefits of UAV based communication, optimal design of UAVs deployment parameters is of fundamental importance.

To efficiently manage the handover due to the movement of $\mathrm{MN}$ and the changing of UAV position, we address the problems associated with UAV selection during handover process to serve the users with the highest possible QoS over many different conditions. The main goal of this article is to present a hybrid UAV selection scheme for VHO management based on UAV-aided wireless communications. In this work, the network selection mechanism during VHO process to enhance the communication of the heterogeneous wireless networks are determined as the multi-objective optimization problem that it can be solved using the UAVs. In recent years, game theory has been used to solve multi-objective design problems, especially for some practical problems in engineering. The game theory is a mathematical model that can makes interaction between rational agents. The game theory used analytical tools to examine the communications of various agents that have conflicting interests [29]. In cooperative games, a set of players has competition to achieve the same goal. Noncooperative game theory [33] deals with how entities cooperate with one another, to reach their own goals. It can be used only in scenarios where agents' interests conflict. The projected technique tries to form this as a decision problem for the MBS which UAV must be selected during handover between UAVs to assist wireless communication. In this respect, game theory offers a technique to solve contradicting situations.Thus, the investigation of actual conflicts via game models in VHO decision is logical. UAVs can be used in a cooperative formation to provide continued connectivity to the existing networks. We try to design a cooperative framework that allowed the formation of a network between the aerial and the ground nodes. Game models are used to explore real conflicts and cooperation processes in the VHO decision process. These key considerations also motivate us to use game theory in our own work. The UAV selection problem is formulated as a trading game-theoretic model.

Recently, the support of mobile drones such as UAV-MNs and UAV-BSs has been explored in 3GPP standardization efforts [34]. Particularly, there has been an increasing interest in the community to characterize the effect of the mobility of drones on their performance, whether they are cellular connected UAVs or aerial BSs [35], [36]. Sharma et al [36] proposed SDN based handover mechanisms to achieve fast MN handover between different aerial cells. Specifically, the UAVs are used as on-demand forwarding switches controlled by the SDN controller. However, there is no strategy for selecting best UAV during handover process. Motivated by this, we develop a UAV-based architecture for best UAV selection during handover process and handover optimization among UAVs. This study is based on utilizing UAVs which can act as self-directed nodes in wireless networking. The offered UAV-based architecture is motivated by [36] but is diverse in procedures as an effective solution for UAV selection and handover problems. UAVs are serving as autonomous nodes that can execute pre-assigned tasks in several applications. The usage of UAVs can be considerably improved with the appearance of small "mini-UAVs" which can increase the benefits in terms of flexibility and cost in connectivity improvement [37], routing [38], and medium access control [39]. A valuable technology for increasing coverage in wireless communication networks is using drone small cells (DSCs) [39]. However, the previous models do not consider UAV selection during vertical handover process.

We present a game-theoretic auction model for communication resources in a handover phase between a mobile node and several UAVs. The UAVs compete with each other to win the cooperation for the source node to connect to it hence forming a procurement auction game. In our business model, we also consider the quality of service requested by the mobile node to the UAVs in terms of the maximum QoS such as maximum bit error probability and the maximum acceptable delay in relaying the data. A cooperative game is designed for the UAV selection problem, and the goal aimed to discover a set of policies for the UAV selection decision in heterogeneous networks. Moreover, competition among different UAVs can satisfy user preferences. User preference plays an important role in the VHO process. The Multiplicative Exponential Weighting (MEW) [40]-[42] is used as the scoring method in the framework of the cooperative computing game. Also, the Analytic Hierarchy Process (AHP) [43] method is applied to weight generation of the UAV selection parameters according to user preference. Furthermore, we have used SDN mechanisms [44] and protocol IEEE (802.21) [35], connected in a single framework as a control plane for the dynamic optimized support of OpenFlow path creation and wireless connectivity formation [17]. The designed scheme built upon the SDN and increments them with MIH [25], [38] services from the IEEE 802.21 standard to maintain seamless mobility across different technologies. Also, we evaluate our model with real data by deriving the data from the publicly available LiveLab dataset which collected in a large Wi-Fi campus network with real users [45].

The main contributions of this paper are summarized as follows. First, an optimization method is proposed to achieve efficient SDN-based network management and fast handovers in UAV networks. Second, the game theory is integrated into heterogeneous wireless networks for solving UAV selection problem during handover process. An adaptive game theorybased model is proposed to select the best UAV during the 
VHO decision stage, between multiple UAVs in the heterogeneous wireless networks. Third, the optimization method is used in this work to design an SDN-MIH-UAV architecture to cope with the limitations of UAVs. The SDN can provide an entire view of the network to formulate end-to-end policies with high computational power that are not available at the UAVs. Forth, an SDN-MIH-UAV architecture is proposed to provide handover facilities in the UAVs with higher throughput and lower handover delay and Signaling overheads. Finally, we obtain SDN-MIH-UAV configurations automatically that outperform the MIH standard one and those used by experts in the current literature.

The rest of the paper is organized as follows. Sec. II formulates $\mathrm{VHO}$ as an optimization problem and illustrates the designed framework. Then, Sec. III describes the architecture of wireless communications with UAVs. Accordingly, Sec. IV explains the design of the proposed scheme, whereas Sec. V qualitatively positions our work with respect to existing literature. Then, Sec. VI discusses the simulation results provided. Sec. VII ends the manuscript with concluding remarks and future directions of research.

\section{Problem Statement}

The problem considered herein is UAV selection during VHO process to support MNs, as using UAVs in the nextgeneration communication systems is a complicated task. Nonetheless, although these aerial vehicles have to deal with some key issues related to their deployment and functionality, they have the ability to manage existing heterogeneous networks and can extend their coverage and capacity [46]. The main drawback is to choose the most effective UAV throughout VHO facility to MN, which is associated with the changing and shifting of its service station. This issue becomes even more complex with (i) high-mobility nodes, $(i i)$ node-density increase and (iii) and application context requirements. Also, scenario $(i)$ can lead to several handover problems like UAV selection among multiple UAVs. The problem should be solved proficiently with a proper solution in order to switch services quickly. The offered technique offers UAV-based handover facilities that can improve the QoS parameters such as latency, signaling overheads, and delay in wireless networks. VHO optimization is a vital process in this study. Fig. 1 displays a designed scenario for a network including UAVs and MBS to assist MNs. This study shows different settings that present the handover signaling for integrated WiFi, WiMAX, and UMTS networks. This figure demonstrates the signaling whereby a $\mathrm{MN}$ is in an overlapped area and can select better connectivity, applying the $\mathrm{ABC}$ concept. The MN under the overlapped area of WiMAX and WiFi is depicted in Fig. 1. Another setting explains the signaling for a user obligated to execute the handover as its current connectivity will be lost as it is moving from WiMAX to UMTS. All scenarios describe how the UAVs can offer service continuity to the user's session as well as some mechanisms involved in these processes.

Hereinafter we explain the main optimization objective, associated to the VHO problem considered.
Specifically, in the VHO decision problem, the objective is to minimize the sum of the signalling overhead, handover latency, number of handovers, and failures in handover.

There is also a payoff game where the aim of maximizing the total reward for the all available UAVs. In order to maximize the total reward of all UAVs, the UAV selection problem can be formulated as Eq. (1). To speed up the convergence of the UAV selection model, as many players as possible should be chosen simultaneously to update their actions in each iteration. However, to avoid the interaction effect between each other, the player and its neighboring MNs shall not be allowed to update their actions simultaneously. Accordingly, we need to first determine the neighboring MNs for each player. We define a local interaction graph to identify the neighboring UAVs for each player. The structure of the proposed local interaction graph is jointly determined by the distance among UAVs and the MNs. In the graph, each node represents a UAV. The problem can be mathematically stated as follows

$$
\begin{aligned}
\max _{P 1} \text { Payoff }_{\text {tot }} & =\sum_{i=1}^{n} u_{i} \times\left(N U_{i}-w_{i} \times N U_{i}^{2}\right), \\
& \underset{\zeta}{\arg \min } \sum_{m \in M} \zeta(m, u),
\end{aligned}
$$

subject to

$$
\begin{aligned}
(\mathrm{C} 1) & : \sum_{i=1}^{n} N U_{i}=1, \\
(\mathrm{C} 2): & \mathbb{P}\left(\sum_{u \in U} \zeta(m, u)\right) \leq P_{u}^{t h} \\
(\mathrm{C} 3): & \sum_{k \in K} \zeta(m, u) \leq 1 \\
(\mathrm{C} 4): & \sum_{k \in K} \zeta(k, u) \leq U_{n} \quad \forall k \in K, \\
(\mathrm{C} 5): & \zeta(m, u) \in\{0,1\}
\end{aligned}
$$

where $\zeta$ is a vector of binary elements $\zeta(m, u)=1$, if mobile node $m$ is chosen to execute an handover to the target UAV $u$, otherwise, $\zeta(m, u)=0$.

The considered objective is subject to a set of different constraints. Specifically, constraint $(\mathrm{C} 1)$ ensures that the payoff for each UAV is the difference between a reward and a cost.

The core goal of the cooperative game is to discover the set of policies which can maximize the payoff function for each candidate UAV, where $u_{i}$ is the current value of utility function of UAV $i$ before accepting the call request and $w_{i}$ denotes the weight on the penalty for unsuitable UAV $i$. The penalty weight is used to avoid the situation that high values are allocated to some unsuitable UAVs. The penalty weight $w_{i}$ is defined to prevent allocating the great preference levels to inappropriate UAVs. Constraint $(\mathrm{C} 2)$ ensures that once MN assigned to a UAV, the probability of handover must be less than a threshold $\mathrm{P}_{u}^{t h}$, determined based on the QoS requirement of the MN's service. The QoS requirement means that effective bandwidth specifies the minimal constant service rate $c$ that 


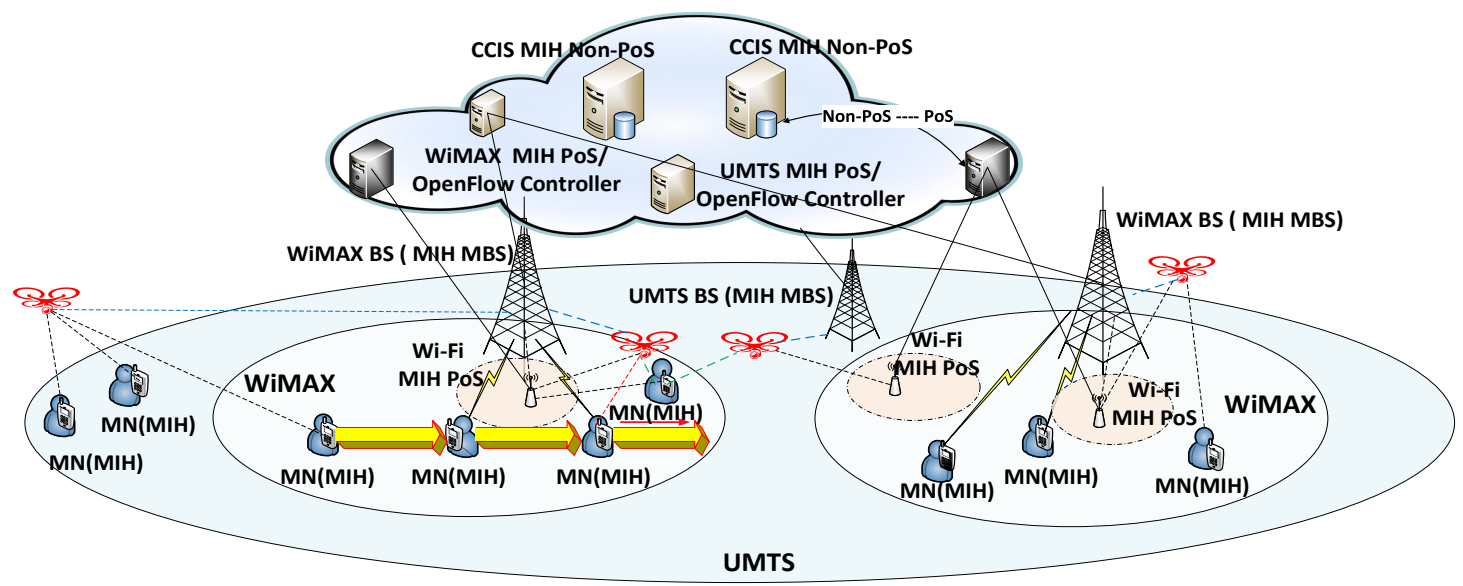

Figure 1. Heterogeneous wireless networks with the coexistence of UAVs and MNs.

can support a given arriving data stream to satisfy the required delay. Also, frequent handovers will increase the overhead and delay in heterogeneous networks. Such frequent handovers will also increase handover failure. The higher number of handovers means higher signalling overhead and hence higher energy consumption. Finally, constraints (C3), (C4), and (C5) indicate that each MN can be assigned to at most one UAV and each BS $\mathrm{k} \in \mathrm{K}$ can serve maximum $\mathrm{U}_{n}$ simultaneously, where $\mathrm{K}$ is a set of BSs.

In general, the value of preference level of any candidate UAV will be greater if the remained available resource of the UAV is higher. Also, preference level for each a candidate UAV will be inferior if the weight of penalty is higher for the UAV. The candidate UAV $i$ will be more appropriate as selected UAV if the mobile node is neighboring to the BS of the candidate UAV $i$. Accordingly, the penalty weight $w_{i}$ is defined in Eq. (8):

$$
w_{i}= \begin{cases}0 & \text { if } d \leq d_{t h} \\ \frac{\left(d_{B, M N}-d_{t h}\right)}{\left(c_{\text {uav }}-c_{t h}\right)} & \text { if } c_{t h}<d_{B, M N} \leq c_{\text {uav }} \\ 1 & \text { if } c_{\text {uav }}<d_{B, M N}\end{cases}
$$

where $d_{B . M N}$ is the distance between the available UAV $i$ and the mobile node, $c_{u a v}$ is the $\mathrm{UAV} i^{\prime}$ cell radius and $d_{t h}$ and $c_{t h}$ are the pre-defined thresholds. When $d_{B, M N}$ is not more than $d_{t h}$, the penalty weight would be zero. In the best case, the goal is to maximize the total reward for the all available UAVs. We have stated the UAV preference game as an optimization problem which displays as Eq.(9):

$$
\begin{gathered}
\max \text { Payoff }\left(N U_{1}, N U_{2}, \ldots, N U_{n}\right) \\
\text { s.t. } \sum_{i=1}^{n} N U_{i}=1 \\
N U_{1}, N U_{2}, N U_{3}, \ldots, N U_{n} \geq 0
\end{gathered}
$$

To solve the problem in Eq. (9), the Karush-Kuhn-Tucker (KKT) [47] conditions are used to find the solution. In the next section, we formulate the $\mathrm{VHO}$ optimization problem as a game theory process.

\section{UAV SELECTION MODEL}

This section describes proposed UAV selection model in detail. To this end, in Sec. III-A we first explain the utility game-theory, whereas in Sec. III-B we describe QoS-based policy for call admission control. Finally, Sec. III-C models the UAV selection problem during VHO from the business perspective.

\section{A. Utility Game-Theory}

The utility-game theory system achieves value for each candidate UAV from the calculations of utility function to improve the QoS and UAV cooperative game. The utility function to improve QoS can compute the utility value according to the QoS improvement requirements of the session request. The cooperative game for UAV selection calculates the value of preference in a multi-UAV viewpoint-based approach. Lastly, the utility value of each candidate UAV is integrated linearly, and the UAV which has the highest value is the best for the session request. The goal of our VHO scheme is to give the $\mathrm{MN}$ the maximum possible QoS profit with the minimum handover cost for any given state. In our model, we use a utility function. For this purpose, a utility function based multiple UAVs deployment model is planned. The designed game-theoretic auction model uses features of UAVs and QoS demands to assign a utility function to each UAV. The utility function is then used to select best UAV during handover process via a game model based on QoS demands. It means that the specification of the best UAV selection is founded on computing the utility function to accomplish the handover task. The best UAV of the set will be the one that needs a minimum amount of fuel with the optimal height and coverage while the power of each selected UAV should be upper than the threshold. Besides, a utility function is used to maintain the QoS through transition among UAVs, which is determined in terms of fuel, height, coverage, and power as well as QoS parameters such as signal interference noise ratio (SINR), latency and received signal power.

We have considered the UAV selection during VHO process as an optimization problem. This challenge addresses the 
tradeoffs involving in a multi-objective optimization problem, where a utility function is to reduce over a multiple-variable. We designed a cooperative game to reach optimal QoS to MNs and reduce the number of handovers. There are three key entities namely game players, strategies, and the payoffs.The set of game players is indicated by $\mathcal{N} \triangleq\{1,2, \ldots, N\}$ where $\mathrm{N}$ is the total number of candidate UAVs, with each UAV following its own policy. We defined the total $n$ strategies, by $\left\{N U_{1}, N U_{2}, \ldots ., N U_{n}\right\}$ where $N U_{i}$ is the value of utility function for $N_{i}, i=1,2, \ldots, n$. Additionally, the total utility function must be minimized. That is, the utility of the current stage using the following equation that is stated as Eq. (10):

$$
\text { Utility Function }=f_{U A V} \times f_{Q o S}
$$

The $f_{\mathrm{UAV}}$ can be divided into two following separate terms as Eq. (11):

$$
f_{\mathrm{UAV}}=f_{\text {len }} \times f_{\text {fuel }} \times f_{\text {cov }} \times f_{\text {pow }} \times f_{\text {hgt }}
$$

First, $f_{\text {len }}$ measures the efficiency of each trajectory, whose functional form is defined as Eq. (12):

$$
f_{\text {len }} \triangleq\left(1-\frac{L_{P 1 P 2}}{d_{0}}\right)
$$

where the actual trajectory length is denoted with $d_{0}$, whereas $L_{P 1 P 2}$ represents the (straight line) distance between the initial and final points $P_{1}$ and $P_{2}$, respectively. Secondly, the term related to the poor quantity of fuel is detailed as Eq. (13):

$$
f_{\text {fuel }} \triangleq \begin{cases}0 ; & \text { if fuel } \text { fuv }_{a v}<\text { fuel }_{d_{0}} \\ 1-\frac{\text { fuel }_{P-P 2}}{\text { fuel }_{d_{0}}} ; & \text { if fuel } \\ a v & \geq \text { fuel }_{d_{0}}\end{cases}
$$

In detail, $f_{\text {fuel }}$ computes the fuel needed for each trajectory and discards each trajectory that needs further fuel than that available at the UAV fuel ${ }_{a v}$. In the above formula, fuel ${ }_{P 1-P 2}$ is the amount of fuel necessary for flying the straight line which connects the initial point $P_{1}$ to the final point $P_{2}$, whereas fuel $d_{0}$ represents the amount of required fuel for flying the actual route.

We calculate the coverage radius of the UAV based on the minimum requirement for the download user coverage probability. The coverage radius is denoted as the maximum radius when the coverage probability for all users located inside the coverage range is more than a stated threshold, $\varepsilon$. The coverage radius for an UAV flying at altitude $h$ and transmitting with a power $P_{\mathrm{UAV}}$, will be given by the following formula Eq. (14):

$$
f_{\text {cov }} \triangleq \max \left\{R: \operatorname{Pr}\left[\operatorname{coverage}\left(\beta, P_{\mathrm{UAV}}, h, R\right)\right] \geq \epsilon\right\}
$$

where $\varepsilon$ is the threshold for the average coverage probability in the area which covered by the UAV and $\beta$ is the SINR threshold. If users are within $R$, then they are considered to be in coverage.

In order to compute the transmit power of UAV, the required power of UAV should be analyzed. We categorized the existing paths into (i) the paths that need extra power $\left(D_{M}=0\right)$, (ii) the paths that no need extra power for traveling $\left(D_{M}>\right.$ $0)$. Herein, $D_{M}$ is the difference between required power and

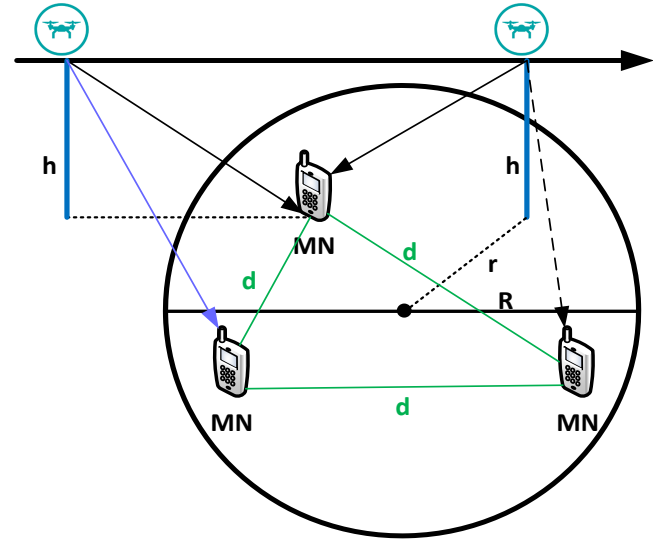

Figure 2. Network model including a UAV at altitude $h$ acting as a flying base station.

available power. If the required power is available for UAV, it can be computed as follows [46]:

$$
P_{\mathrm{UAV}}=\frac{1}{1+C \exp (-B[\theta-C])}
$$

where $P_{\mathrm{UAV}}$ is the transmit power of $\mathrm{UAV}, C$ and $B$ are constant values depending on the type of environmental technologies in rural and urban environment, and $\theta$ is the elevation angle. Considering that the required power is not available, there is a penalty constant $P C$.

The power objective is determined in Eq. (16) as follows:

$$
f_{\text {pow }} \triangleq \begin{cases}P_{\mathrm{UAV}} ; & \text { if } D_{M} \leq 0 \\ P C+\frac{D_{M}}{d_{0}} & \text { if } D_{M}>0\end{cases}
$$

where $d_{0}$ is actual path distance of the UAV, while $P C$ is a penalty constant.

Considering a Line-of-Sight (LoS) link, the received signal power at $j$ th UAV from $i$ th device is expressed by Eq. (17) (in $\mathrm{dB}$ ):

$$
P_{r, i}^{j}=P_{t, i}-10 \alpha \log \left(\frac{4 \pi f_{c}}{c} d_{i j}\right)-\eta
$$

where $P_{t, i}$ denotes the transmitted signal power from $i$ th device (in $\mathrm{dB}$ ) at the time slot $t, f_{c}$ is the carrier frequency, $\alpha=2$ is the path loss exponent for LoS propagation, $\eta$ is the excess path loss added to the free space propagation loss, $d_{i j}$ is path length from $i$ th device to $j$ th UAV and $c$ is the speed of light.

Consider a circular area with a radius $R$ in which a number of wireless nodes are deployed. In this area, UAVs at altitude $h$ are deployed to act as flying base stations for serving those users. The UAV flying height is associated with communication range $R$ (Fig. 2). The maximum altitude $h$ of UAV from sensor node within the maximum communication range can be calculated from Eq. (18). The term is related to the height of the path is described as follows:

$$
f_{\mathrm{hgt}} \triangleq \sqrt{R^{2}-\left(\frac{v T_{c}}{2}\right)^{2}}
$$

where $v$ and $T_{C}$ are the UAV speed and data exchange time, respectively. Typical average and maximum values for $v$ are 
$15 \mathrm{~m} / \mathrm{s}$ and $20 \mathrm{~m} / \mathrm{s}$, respectively. For a UAV speed of $15 \mathrm{~m} / \mathrm{s}$ (resp. $20 \mathrm{~m} / \mathrm{s}$ ), typical values of $T_{C}$ lie within $1: 90 \mathrm{~ms}$ (resp. $16: 28 \mathrm{~ms})$.

Considering that in a network, a receiver could successfully receive data only if the SINR is higher than a threshold value.

The explanation of computing the QoS criteria are mentioned in the following in Eq. (19) as:

$$
f_{\mathrm{QOS}} \triangleq\left(f_{\mathrm{SINR}_{\mathrm{MN}-\mathrm{UAV}}}+f_{\mathrm{RSP}}\right)
$$

For each MN that can connect to the UAV, the SINR is measured in Eq. (20) as:

$$
f_{\mathrm{SINR}_{\mathrm{MN}-\mathrm{UAV}}} \triangleq \frac{P_{\mathrm{UAV}}}{I_{t}+N_{0} \times W}
$$

where $P_{\mathrm{UAV}}$ denotes the received signal power from the $\mathrm{UAV}, N_{0}$ denotes the noise power, $W$ is the system bandwidth, and $I_{t}$ the overall interference power from (other) transmitters.

The received signal power $\left(f_{\mathrm{RSP}}\right)$ at each user location depends on LoS or Non-Line-of-Sight (NLoS) condition between the user and UAV and it can be computed by [46] in Eq. (21) as:

$$
f_{\mathrm{RSP}} \triangleq \begin{cases}P_{\mathrm{UAV}} d_{\mathrm{UAV}}^{-\alpha_{\mathrm{UAV}}} & (\mathrm{LoS}) \\ \beta P_{\mathrm{UAV}} d_{\mathrm{UAV}}^{-\alpha \mathrm{UAV}} & (\mathrm{NLoS})\end{cases}
$$

where $d_{\mathrm{UAV}}=\sqrt{r^{2}+h^{2}}$ is the distance among a user and the $\mathrm{UAV}, \alpha_{\mathrm{UAV}}$ is the path loss exponent on the user-UAV link, and $\beta$ is a further attenuation factor due to NLoS connection.

In the case of handover, the SDN controller should select a UAV which guarantees optimal QoS to MN. There will be some options that can undertake this task and it requires applying an efficient selection mechanism. The information about candidate UAVs will be sent to SDN which should be a combination of these UAVs. After making the possible set of UAVs, the best one should be selected. We employ MEW as a scoring technique in the cooperative game model. The MEW is a multiple attribute decision making scoring technique [42]. The score function is built on different criteria such as length, fuel, SINR, and so on. In this model, the AHP method is applied to compute the weight of the network selection parameters based on user preference [43]. At the end, the decision is made based on the user's preferences and various criteria parameters. When the SDN controller selects a UAV, an agreement is made with the UAV. The network selection problem is considered as a trading market competing game model. The game players are the UAVs, the policies are the reaction of game players for consideration of user's preferences. The user's preferences expressed in the weight of the parameters. Also, the payoff depends on the UAV's capability to meet the user demand. SDN controller continuously programmed by the control plane to meet the requirements of the current context.

Fig. 3 displays the proposed UAV selection model. In this model, the decision that a user might take could be either to stay in the current network or to move to another available network that could provide a better service. Whenever one of the events mentioned above occurs, the UAV selection model is executed to choose the best UAV that can provide the required service before the handover execution process occurs. In this model, the UAVs firstly start to send their offers to the controller. Then, the controller should evaluate the ability of the UAVs based on initial offers. So, the controller can recognize the ability of each UAV to provide better offers. After that, the controller should start to negotiate with the UAVs by distributing user's preferences. Then, the UAVs are reacting to user's preference based on their strategies. The strategies are appointed as the weights of the parameters which have high effect on handover decision making. In continue, each UAV should evaluate and send its own final offers based on the user's preference. Then the controller finds the best offer among the final offers using the weighted matrix. In the last stage, the best UAV which can offer maximum utility function score is selected as a winner.

The UAV selection problem during VHO can be summarized as a matrix where $i$ th row corresponds to $i$ th UAV candidate whereas $j$ th column corresponds to one of the considered metrics (e.g., length, fuel). The score $S_{i}$ of $i$ th UAV is obtained as the weighted product of all the metrics considered Eq. (22), namely:

$$
S_{i} \triangleq \prod_{j=1}^{N} x_{i j} w_{j}
$$

where $x_{i j}$ indicates $j$ th metric of $i$ th UAV, whereas $w_{j}$ represents the (importance) weight assigned to $j$ th metric in the score evaluation, with the weight set forming a convex combination (i.e. $\sum_{j=1}^{N} w_{j}=1$ ). Note that in Eq. (22), $w_{j}>0$ (resp. $w_{j}<0$ ) for benefit (resp. cost) metrics.

Since each UAV score obtained through MEW is not upperbounded, it is convenient to measure and compare it with the score that would be achieved by an ideal UAV.

More specifically, the best UAV is determined as the UAV with the highest score. The score function is used to calculate the score for each UAV.

The best value is the lowest for a cost metric. Accordingly, we define the value ratio $r_{i j}$ between $i$ th UAV and the ideal $\mathrm{UAV}$ as Eq. (23):

$$
r_{i j}=\frac{U A V_{i}}{U A V^{*}}=\frac{\prod_{j=1}^{N} x_{i j}^{j}}{\prod_{j=1}^{N}\left(x_{i j}^{*}\right)^{w_{j}}}
$$

where $0 \leq r_{i j} \leq 1$

The total score of the main offer for each UAV is acquired as presented in Eq. (24):

$$
U_{\text {primary }}^{i}=\sum_{j=1}^{m} r_{i j}
$$

The core role of the data fusion unit is to analyze, compute, and fuse the raw data to arrange for an accurate weight of the attributes. The weighted matrix can be computed by AHP technique to achieve the relative weight $w_{j}$ of the $j^{t h}$ attribute in Eq. (25) as:

$$
\sum_{j=1}^{m} w_{j}=w_{l}+w_{f}+w_{c}+w_{p}+w_{h}+w_{s}+w_{r s}
$$




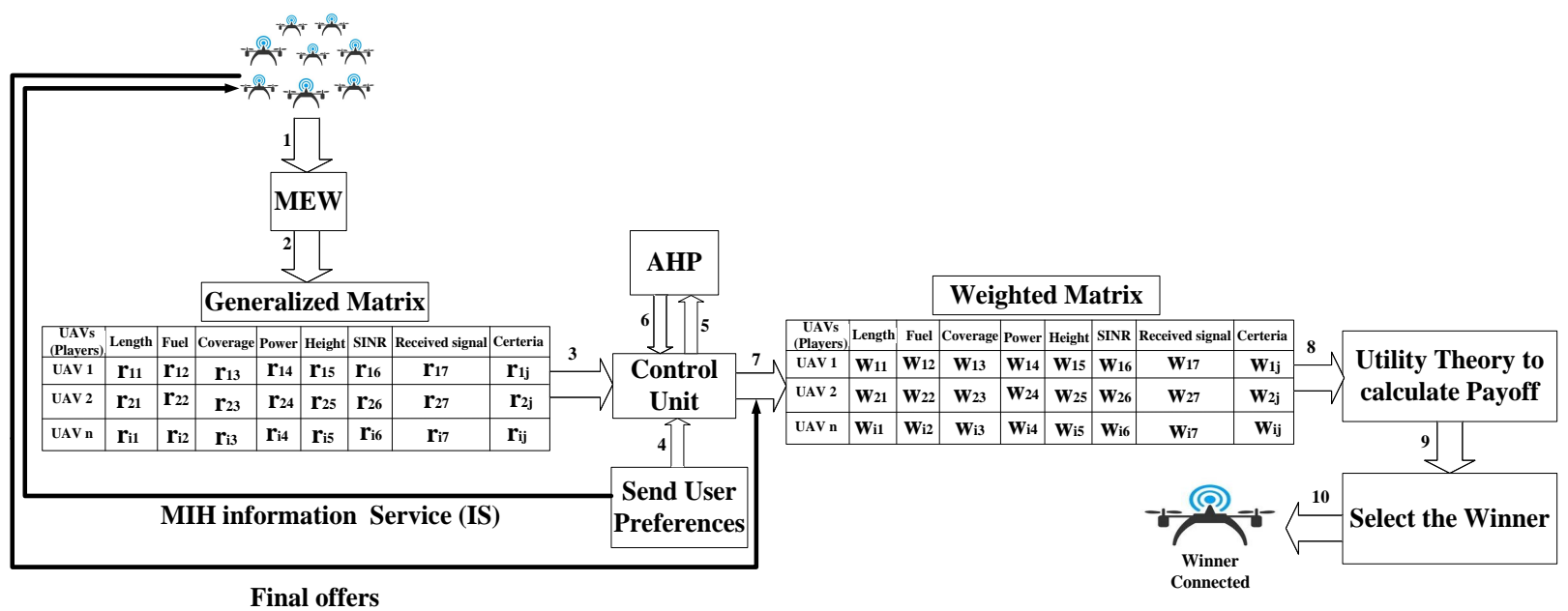

Figure 3. Steps of UAV selection model based on game theory to choose the best UAV.

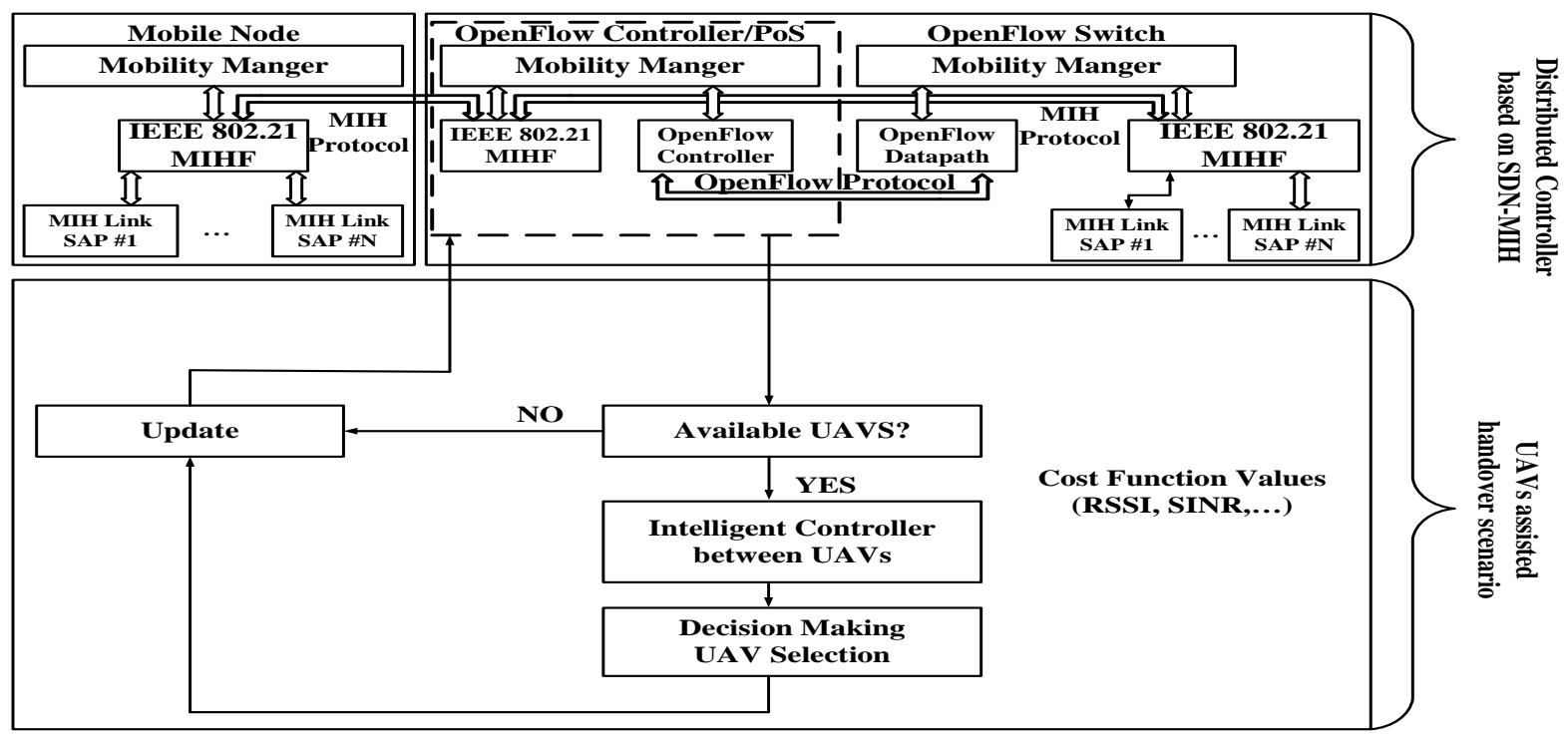

Figure 4. State diagram for SDN-MIH-UAV control plane.

where $w_{l}, w_{f}, w_{c}, w_{p}, w_{h}, w_{s}, w_{s}, w_{r s}$ denote the weight or importance of the metrics which have effects on the UAV selection procedure, namely, length, fuel, coverage, power, height, SINR and received signal respectively.

Game utility value $U_{i}$ for the $i^{\text {th }} \mathrm{UAV}$ is synthesized. In independent metrics, the value of $U$ is the product of multiplying relative user preference weight $w_{j}$ with nondimensional comparable data $r_{i j}$ in Eq. (26) as follows:

$$
U^{i}=\sum_{j=1}^{m} w_{j} r_{i}
$$

where $U^{i}$ is the total utility for candidate $U A V_{i}$.

The utility (score) function is calculated for each UAV. Accordingly, the UAV with the highest score is selected as the preferred $\mathrm{UAV}$, namely $\mathrm{UAV}^{\star}$ is the $\left(i^{\star}\right)$ th $\mathrm{UAV}$ such that:

$$
i^{\star} \triangleq \arg \max _{i} U^{i}
$$

\section{B. Call Admission Control}

The call admission is the decision to accept or reject a new call. It is needed to design a call admission control that maximizes the network utilization while supporting various services for multiservice wireless networks. The goal of this section is to minimize the sum of call blocking probabilities. The criterion should be given to the handover calls over new calls by the call admission policy. We used the QoS-based policy for call admission control. We assumed that each user has a rate requirement $\mathrm{R}_{i}$. The call admission policy checks the bandwidth when a new call arrives if bandwidth is available it connects the call otherwise it terminates the call. For the QoS guarantee of user $i$, the transmission bandwidth of $U A V_{j}$ is determined by

$$
B_{i j}^{U}=\frac{R_{i}}{\log _{2}\left(1+\operatorname{SINR}_{i}\right)}
$$


Each UAV shares a proportion of the resource defined as $\alpha_{u}$ with macro users and $\left(1-\alpha_{u}\right)$ is allocated to users of UAV. The achievable bandwidth allocation needs to be more than the bandwidth requirement of each user, as follows:

$$
\frac{\left(1-\alpha_{u}\right) \cdot \mathrm{B}_{u}}{\mathrm{~N}_{u_{i}}(t)} \geq \max B_{i j}^{U}
$$

where $\mathrm{B}_{u}$ is the available bandwidth of $\mathrm{U} A V_{j}$ and $\mathrm{N}_{u_{i}}(t)$ is the number of users served by $\mathrm{U} A V_{j}$. In order to QoS guarantee of $\alpha_{u}$, the criterion of admission control is given as follows:

$$
\frac{\max \mathrm{B}_{i j}^{U} \cdot \mathrm{N}_{u_{i}}(t)}{\mathrm{B}_{u}} \leq 1
$$

The users who can meet the criterion (29) will be accepted by UAV and other users should serve by MBS.

\section{Business Model}

Different from the existing studies, in our study, we model the UAV selection during VHO problem as an optimization problem from the business perspective, aiming to maximize the profits of the MNs. We present a game-theoretic auction model for communication resources in a handover phase between a mobile node and several UAVs. The UAVs compete to win the cooperation for the source node to connect to it hence forming a procurement auction game. In our business model, we also consider the quality of service requested by the mobile node to the UAVs in terms of the maximum QoS such as maximum bit error probability and the maximum acceptable delay in relaying the data. In our cooperation model, the $\mathrm{MN}$ in order to keep connection to an access network especially when the QoS is low tries to cooperate with UAVs to reach optimal QoS during vertical handover process. The MN broadcasts a cooperation-request with the destination details and the required QoS for available UAVs. The UAVs respond to the request with the price quotations in terms of $\beta_{i}$ (Euros/bit) with the offered QoS. The QoS requirement at $\mathrm{U} A V_{i}$ is converted to an equivalent cost, priced as $\beta_{i}$ (Euros/bit). We assume that for all UAVs and MNs, there is a fixed common cost $\mathrm{C}$ (Euros/bit) known to all nodes in the network. The price $\beta_{i}$ quoted to $\mathrm{MN}$ by $\mathrm{U} A V_{i}$ is defined as follows:

$$
\beta_{i}=\mathrm{C} . \mathrm{U}^{i}
$$

where $\mathrm{U}^{i}$ is the total utility for candidate $\mathrm{U} A V_{i}$. The $\mathrm{U}^{i}$ is a real number always greater than one known as the pricing index which is decided by $\mathrm{U} A V_{i}$. When $\mathrm{U} A V_{i}=1$ the $i^{t h}$ UAV gains no profit by helping $\mathrm{MN}$ but simply covers the cost for the cooperation. When $\mathrm{U} A V_{i}>1$ a profit of $\mathrm{C}\left(\mathrm{U} A V_{i}-1\right)$ (Euros/bit) is made by the respective UAV. In our model, we assume that each $\mathrm{U} A V_{i}$ has a minimum value for $\mathrm{U}^{i}$ defined as $1<\mathrm{U}_{\text {min }} i \leq \mathrm{U}^{i}$ that gains a minimum profit for the cooperation if it wins. The determination of $\mathrm{U}_{i}$ min is modeled as a utility function Eq. (26). The computation of $\mathrm{U}_{i}$ min is described in detail in this section. An interesting feature that could be adopted in the business model is the 'businessmode' compared to the 'friendly-mode' feature. In the business model, $\mathrm{U}^{i}$ will have a motivation to make economic profits, and in the friendly-mode feature, the cooperating $\mathrm{U}^{i}$ can

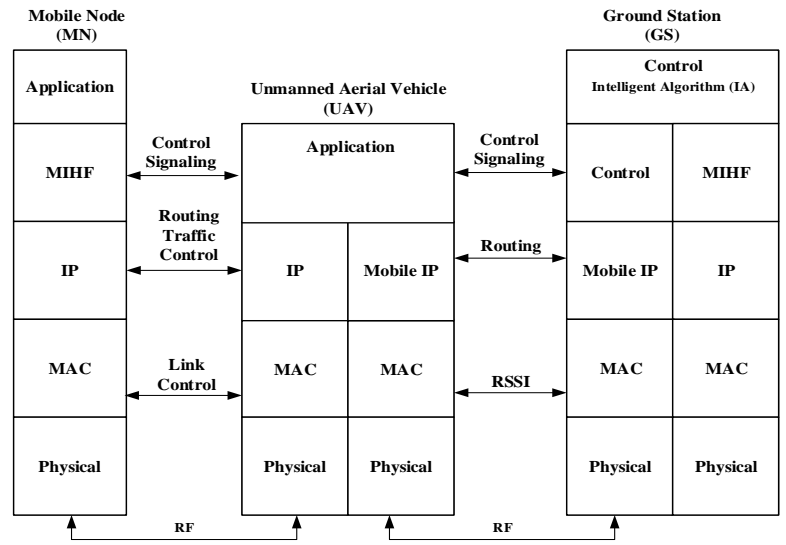

Figure 5. A two-hop layer model among UAV, MN and MBS.

choose to help a friendly node without being motivated by any economic gains as discussed in this paper. The MN therefore could choose between the two options for VHO process. This model can be a sufficient solution for UAV selection during the VHO process between multiple UAVs. The goal of proposed model is to give the MN the maximum possible QoS profit with the minimum handover cost for any given state.

\section{SCHEME DESIGN}

Th architectural design contains all the design requirements of proposed model. In Sec. III-A we first explain the distributed controller based on SDN-MIH, whereas in Sec. III-B we describe the two-hop model designed in this work. Finally, Sec. III-C explains the detailed procedures for the handovers Between UAVs.

\section{A. Distributed Controller Based on SDN-MIH}

The policy controller for the offered method and handover process with state diagram as shown in Fig. 4. The controller continuously monitors the network load and checks for its capacity to handle it. In the case of demands for UAVs, the UAV selection model is invoked, which takes care of policies in the UAV control plane and initiates it. Now the SDN shifts from regular operations to SDN-MIH mode, and network services are invoked with other management policies depending on the features such as charging and energy consumption especially for controlling the UAVs.

We use distributed mechanisms in nodes configuration and paths establishment via SDN controller [39] for dynamic mobility management. Also, we call IEEE 802.21 Media Independent Handover [38] services to facilitate handovers and triggers from link layers in heterogeneous wireless environment. This standard offers link-layer intelligence and other associated network information to higher layers to optimize handovers among heterogeneous networks.

We need to design an efficient and robust end-to-end data relaying between UAVs, MNs and base station. In this context, we offer an aerial network management protocol constructed on top of an SDN architecture. In our network model, each UAV converts to an SDN switch which acts under commands 
sent by a centralized controller [36]. Also, the combination of SDN and MIH leads to improved connectivity. Because it offers best connectivity among available candidates, OpenFlow procedures can be triggered to prevent traffic disruption by standards compliant nodes. The designed controller should monitor the load on the network. The controller constantly checks the capacity to reduce network load constantly. During vertical handover process, in competing demands for UAVs, the UAV selection method should be called and initiated. Then, the SDN shifts to SDN-MIH-UAV mode by enabling the interrupt service to pause regular operations. In our framework, the MIH allows the defined mechanisms to deploy over all link technology. The MIH can collect real-time information about current link status such as resource availability check, preparation, commit and release, as well as receiving events perceived by the MN. Hence, Open Flow can use the QoS information of MIH in Layer 2 and then select the best UAV as optimal handover candidate based on UAV and the MN's connectivity to optimize the usage of network resources. In continue, other procedures are called for managing the network services based on the fresh reachability tables that governs controller behavior. Once the MN handovers to the new UAV, the OpenFlow path is already established. Then, MIH can facilitate IP mobility procedures with its flexibility to integrate with different mobility protocols. In our SDN-MIH based framework, we have assumed that there are terminals to UAVs and MBSs. As, MNs play the role of terminals for UAVs and also the UAVs play the role of air terminals to MBSs. We have considered a control plane to handle and manage the traffic flowing procedures with a low-complexity model, computation time and cost.

The main component of the proposed framework is the "OpenFlow Controller/PoS". This controller not only performs routing tasks, but also controls and handles mobility procedures. It means that OpenFlow Controller should update forward tables of OpenFlow Switches as forwarding elements. The proposed OpenFlow Switch/PoA has same performance with the standard Open-Flow switch in performing data packet forwarding operations. It has a flow table for storing information of each flow for configuration via the OpenFlow protocol. The module of mobility manager involves the OpenFlow Controller of the IEEE 802.21's point of service (PoS) that includes a MIHF for exchanging IEEE 802.21 with other nodes. Generally, the proposed OpenFlow Switch/PoA acts as a PoA because it can provide link connectivity to a $\mathrm{MN}$. Also, the module of mobility manager combines the activities of OpenFlow and MIH to control parts of the link interface about handover management.

\section{B. Two-Hop Model}

A two-hop model is designed in this work that is shown in Fig. 5. The first hop is from the MBS to the UAV, the second hop is from the UAV to the user terminals. In the proposed UAS-R system, there is no need to equip a large number of BSs near the trackside. Instead, only a few MBSs far away from the tracks are required. The proposed model can significantly mitigate the Doppler Effect, the multipath effect, and the penetration loss simultaneously.
In the airborne peer-to-peer networking architecture, each UAV acts as a relay node to receive and forward data. Communications between a UAV and a MBS or a terminal can take place over several hops through intermediate UAVs. The open space simplifies the connection supplies, and the bandwidth can be used more regularly and efficiently. Besides, the nodes are mobile in order to support potential communications.

The cross-layer handover is projected to give the MT services that are seamless in the heterogeneous networks that are wireless. In reducing the time of delay in handovers, the layer links should initiate the protocols for handovers of the layer 3 at proper times; hence, the process can be completed before end of the time of the current wireless connection. A cross-layer construction is introduced in the proposed layer model, where the collaboration between the first three layers of OSI reference model is facilitated. Cross-layer design builds on the sharing of information between the first three layers. It is revealed that based on the UAV attitude variations (pitch, roll and yaw); the performance of the designed handover management scheme can be enhanced by the help of this crosslayer construction.

In the UAV-to-mobile node (U2MN) communications, the UAVs act as airborne relays between the MNs and the MBSs to provide high-speed data links and seamless connectivity for mobile users. The U2MBS and U2MN channels share many characteristics, such as less shadowing effect, less multi-path effect, and LoS or NLoS dominates the channel [46]. The UAV selection allows an efficient deployment of UAVs. Only the optimal UAV is selected based on defined utility function which guarantees optimal QoS to MNs. Thus, the selected UAV can offer optimal QoS to MNs. However, there is a constraint, while considering the coverage scenario. Since the coverage requires a shift of services from UAVs to UAVs; this raises an important issue of handovers in the UAVs-assisted networks. This leads to inclusion of new or existing handover features to resolve communication overheads related to the shift of services. This will certainly affect the service timings, which may cause extra delays in the network. Also, extra communication equipment has to be added to resolve this issue. During the handover process, the control information must be transmitted through layer 3 (L3) in SDN and also the L1, L2 layers are used to synchronize the data from the network components in the network [25].

\section{Intelligent Handoff Decision between UAVs}

The UAV to UAV (U2U) channels are viewed as free space channels based on the assumption that a dominant LOS path is available. When two MNs are running in opposite directions on adjacent tracks respectively or are running close in the same track, two UAVs may fly near each other. The UAVs can gain many benefits, e.g. collision avoidance in the UAV to UAV communications. They exchange information about position, altitude, recently identified ground obstacles, and flight path from each other. Also, the UAV to UAV communications can help to control the mobile user operation to avoid possible accidents like handover failure with the help of the ground station control centralized $r$. The idea of 
this assistance is that a source UAV (associated with source network) uses another UAV (associated with target network) within its communication range as a relay to transmit signals to the target network on the same track. Additional UAV to UAV links are used for relaying those UAVs that are during an outage or a connection failure to ground stations within the communication range. Besides, the UAV to UAV links also allow both control data and user data to get routed through the UAV to UAV distributed network.

Let's assume that multiple UAVs are flying above heterogeneous wireless networks and each UAV is planned for a specific task [28]. The SDN controller [36] not only manages activities of multiple UAVs but also receives requests for UAVs to do vertical handovers in certain regions. The vertical handover refers the handover across two radio coverage cells from diverse networks [48]. We used UAVs as aerial access points through their operations during vertical handover process. In this study, the vertical handover management refers to arranging policy that improves session maintenance of aerial nodes among the network nodes. In this case, the controller of SDN has to make a decision in which UAV should be the best option during handover process in a smart manner. This smart UAV selection significantly depends on the equipment onboard as well as power level, the time required to complete the vertical handover process, distance, and the speed of the UAV. Studying these parameters is essential in fulfilling efficient vertical handover management successfully.

The sequencing diagram showing a network that comprises of UAVs and MBS to serve MNs during the handover process in Fig. 6. In the scenario, after the $\mathrm{MN}$ determines the handover to a new available network due to loss of signal quality or users' preference, the MN can activate the mechanism MIH_Get_Information Request to obtain information simultaneously about the CRANs around the MN as well as the resources available in every one of them. Our proposed model, which is the OpenFlow Switch/PoA, has updated realtime information on the radio resources available in every underlying RAN. As a result, the OpenFlow Switch/PoA can update its database with the new resource information.

Then, the source node (UAV-1) sends the initial measurement to the requesting MN. In continue, the OpenFlow controller should select the best UAV based on received realtime information from source UAV and other available UAVs in a certain regions of network. According to characteristics of the dynamic UAV, the UL allocation link should be initiated from the current base station after the completion of the handover decision process. In response to this event, the MN reports real-time data such as the received signal strength indicator (RSSI) towards the current MBS. After that, the controller of source node is responsible to send the handover request to the current MBS's controller. The controller will forward the request of handover to the UAV2 as a target node. The controller of target MBS receives the notification from selected node. Then, the controller of the target node will send the handover acknowledgment. The controller of current MBS should initiate a downlink allocation signal from MN by the source node. The downlink allocation will be tracked via a handover command. In continue, the controller will transfer the real-time information of the $\mathrm{MN}$ which has moved towards the selected controller for handover initiation. The MN synchronizes the connection with the target UAV after acquiring the signal. Then, the uplink allocation of MN will acknowledge this synchronization in order to acquire handover confirmation from MN. In the end, the selected node as a target point will confirm the handover process with its controller by sending an acknowledgement to the controller of the current node for handover while its table should be updated.

\section{Comparison with the State of the ART}

We have performed a qualitative comparison to prove the novelty of the proposed model. In detail, the comparison with the state of the art is summarized via Tab. I. The aforementioned table lists some recent models using UAVs as flying wireless communication platforms as well as those that have utilized SDN for network management and handovers between the moving nodes. There are some works which can manage handover problems [26], [27]. They proposed SDN based UAV architectures. However, there are no policies designed specifically to select the best target in VHO process. Some architectures for collaborative UAVs have developed [21], [22], [23]. These models mainly focused on UAVs deployment for capacity, and network coverage improvement. Most of the works [21], [25], [26], [27], [28] have designed for network monitoring by assistance from SDN controller. Despite all advantages, the controller in SDN is centralized and can lead to concerns about SDN scalability and resilience. In the centralized methods, all the nodes should be in LoS environments to support a moving $\mathrm{MN}$ and to success of handovers between the aerial nodes. The distributed controller can provide easy troubleshooting of a network. These controllers provide high access to dynamic access points. In our proposed model, the controller is distributed as opposed to all related studies. Thus, handover procedure can be completed when UAVs have covered their area and coming from LoS environment. This advantage can lead to a latency reduction in comparison to the centralized methods.

\section{Numerical Evaluation}

The objective of this section is to numerically evaluate the proposed approach and compare it with existing alternatives. To this end, in Sec. VI-A we first detail the simulation setup considered herein, whereas in Sec. VI-B we introduce the relevant performance metrics adopted for comparison. Finally, Sec. VI-C reports the numerical results and the corresponding discussion.

\section{A. Simulation Setup}

We used a dataset from Rice University LiveLab [45] to assess the proposed approach. LiveLab is a methodology to measure and analyze real-world smartphone usage and wireless networks designed for long-term user studies. The LiveLab database is provided in a collection of SQL files and divided based on dataset features. Features of the dataset include the applications running in the terminals and the set of available access points to each terminal at a given 


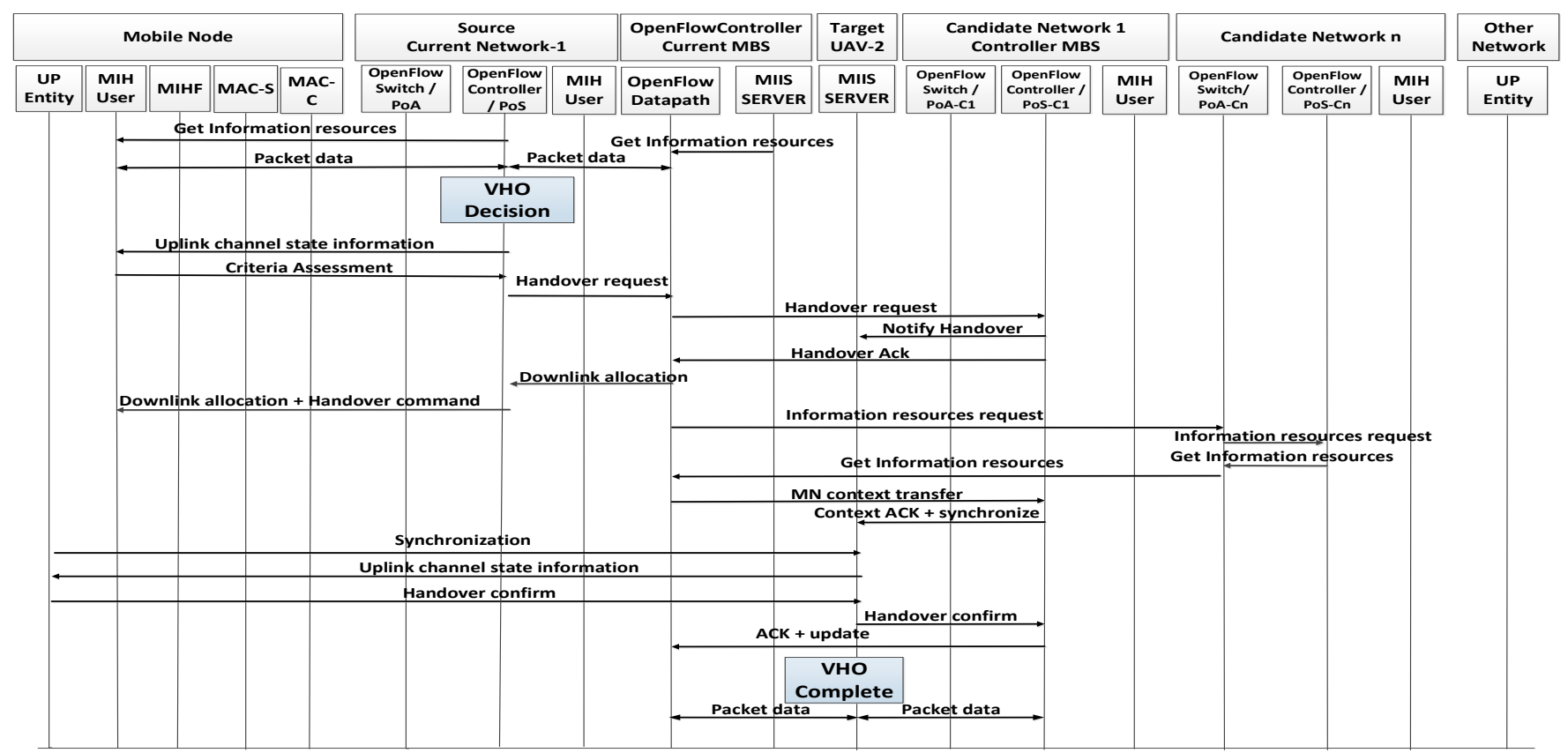

Figure 6. Detailed procedures for the handovers between UAVs.

Table I

COMPARISON WITH THE STATE OF THE ART.

\begin{tabular}{|c|c|c|c|c|c|}
\hline Model & Handover support & UAVs support & Network Mgt & Controller & Scalability \\
\hline Sara et al. [21] & 0 & - & - & Centralized & Medium \\
\hline Merwaday et al. [22] & 0 & 0 & 0 & - & High \\
\hline Fadlullah et al. [23] & 0 & $\bullet$ & 0 & - & High \\
\hline Kim et Feamster [25] & 0 & 0 & 0 & Centralized & Medium \\
\hline Yang et al. [26] & • & 0 & • & Centralized & High \\
\hline Iqbal et al. [27] & - & 0 & • & Centralized & High \\
\hline Sharma et al. [28] & 0 & • & • & Centralized & High \\
\hline Proposed model & - & • & - & Distributed & High \\
\hline
\end{tabular}

Table II

Selected Rice Dataset Features.

\begin{tabular}{lll}
\hline Filename & Trigger & Description \\
\hline appusage.sql & user event & applications run by users \\
Ucelltower.sql & periodic & cell tower connected \\
availablewifi.sql & periodic & available wifi access points \\
apps.sql & - & list of all installed applications \\
call.sql & event & phone calls made received by users \\
sleep.sql & event & spent time in low power mode \\
display.sql & event & display status \\
charging.sql & event & charging state of phone \\
web.sql & event & web browsing history \\
loggeron.sql & event & time that the logger was running \\
cellsignal.sql & periodic & cell signal strength \\
iostat.sql & periodic & cpu and disk utilization \\
accel.sql & periodic & accelerometer readings \\
power detail.sql & event & built-in logfile driven \\
associatedwifi.sql & periodic & connection to the associated AP \\
\hline
\end{tabular}

time. Selected dataset features described in Table II. Some experiments were also conducted by re-introducing the day of the month feature along with 'time' (of day in seconds) to observe any performance differences for the RICE dataset. We classified the applications of LiveLab dataset into different classes: video streaming, calls, messaging, e-mail, sharing and synchronization, entertainment, and social applications. Then,
Table III

Application Categories for Livelab DataSet.

\begin{tabular}{lll}
\hline Class & Tag & Average Rate \\
\hline Social media without audio & small & $12.50 \mathrm{Kbps}$ \\
Sports & small & $17.50 \mathrm{Kbps}$ \\
VoIP (Skype) & small & $16.50 \mathrm{Kbps}$ \\
Sharing (Dropbox) & small & $12.50 \mathrm{Kbps}$ \\
Email & small & $12.50 \mathrm{Kbps}$ \\
News & small & $43.50 \mathrm{Kbps}$ \\
Social media with audio & small & $45.50 \mathrm{Kbps}$ \\
Video streaming & big & $2.50 \mathrm{Mbps}$ \\
\hline
\end{tabular}

we tagged each class as "small" or "big" depending on the average demanded bandwidth. Table III indicates the average rates and tags of classes.

The proposed framework is assessed by using the wellknown Mininet network emulator with OpenFlow, allowing SDN-based testing through virtual environments [49]. Mininet contains OpenFlow controller, virtual host, and OpenFlow switch. The control plane of proposed scheme is divided into network control plane and UAV control plane. The network control plane which is connected to the data plane forms the plans to manage the flow, topology and routing. The UAV control plane mainly controls the topology, configurations, and logs related to UAVs. The flow table includes three fields 
namely flow, data, and log. The flow includes source and destination address, target node, session id,and port no. The data express traffic rate, network load and active users. The logs display UAVs route, battery info, location, total load, up time, charge and, sub-clients.

We have evaluated our suggested methodology over an area of $10 \mathrm{~km}^{2}$, handled by a maximum of $12 \mathrm{UAVs}$ per MBS using the numerical simulations. The MNs are chosen a new location and moves to the chosen location in per milliseconds in random waypoint mobility. However, the UAVs are organized for referencing the $\mathrm{MN}$ mobility model. There are three access points (APs) with $200 \mathrm{~m}$ radio range and initial rate of $256 \mathrm{~kb} / \mathrm{s}$ around each cell. Also, there are the mean of 300 users which follows a Poisson distribution of mobile users over the network. The results are recorded in terms of different methods via the standard SDN-UAV network architecture. The designed model is modified for SDN-MIH-UAV networks with UMTS, Wi-Fi, and WiMAX PoA in the various areas with different data rates offered.

The growth of MNs requesting handovers at the same time which affects the performance of the proposed framework. The other relevant setup parameters to evaluate the performance of our framework are reported in Tab IV.

We have presented our evaluation with an operating time of $1000 \mathrm{~s}$ during 60 runs, in order to handle the service requests of users. Since the UAV altitude $h$ affects the quality of service and quality of experience to the $\mathrm{MNs}$, in our scenario we change $h$ within $[250,550] \mathrm{ft}$. Our simulation experiments are detailed for the QoS parameters such as ( $i$ ) packet delay, (ii) throughput and (iii) latency [49], which are compared with a similar method [36] and the SDN-UAV architecture, the designed SDN-MIH-UAV architecture within UMTS, Wi-Fi, and WiMAX PoA in heterogeneous environment.

\section{B. Performance Metrics}

Simulation results presented that choosing the AHP and game theory can reduce the utility function and the proposed framework can prevent the amount of unnecessary ping-pong handovers. The performance results show that our framework with increasing the MNs can reduce latency during session transferring in handovers in comparison with original SDN.

In case of Eq. (32) by [49], the handover latency is the time length between the handover initiation and the handover completion, once handover decision is executed.

$$
\operatorname{lat}_{\mathrm{HO}} \triangleq(1-\sigma)\left(f_{t}+w_{t}\right)+\sigma h_{t}
$$

where $h_{t}$ is the handover time of the $\mathrm{MN}, f_{t}$ is the revision time on a radio link failure, $w_{t}$ is the waiting time in a handover making and $\sigma$ is the probability of successful handover.

Then, we calculated the end-to-end delay based on connection delays such as propagation delay, packet transmission delay, queuing delay, and processing delay of the network in handover of the UAVs to another ground station in UAV payloads.

The latency impacts the end-to-end delay and influences it with a similar variation.
Handover delay is contained of several elements including Link and IP layer handover latencies, and packet delivery delay. It should be mentioned that the period of packet buffering and binding update is equal to the time taken to perform link and IP handovers, and the packet forwarding period.

The calculation of the overall mean delay for completing the VHO preparation phase involves various delay components, which will be enumerated in different components of delay. Specifically, the total delay of the proposed MIH-based vertical handover $D_{\text {total }}$ can be expressed as Eq. (34) by [28], [39]:

$$
D_{\text {total }} \triangleq D_{h o}+D_{L 2}+D_{H L}
$$

where $D_{h o}$ is the handover delay across different access network technologies, while $D_{L_{2}}$ is the delay of handover layer 2 and $D_{H L}$ delay of higher-layers handover. The term $D_{h o}$ is mainly due to delays caused by handover initiation, selection and preparation procedures while a handover is performed.

$$
D_{h o} \triangleq D_{I}+D_{P}+D_{S}
$$

Accordingly, in the above formula, $D_{I}$ and $D_{P}$ are the delays of handover initiation and preparation, respectively. On the other hand, $D_{S}$ denotes the delay of network selection phase during the handover.

As a major problem, a large number of connected users make the impractical handshaking-based access control, due to the high communication overhead. As a benefit of the proposed model, users can instantly transmit their signals to a base station in any available time slots without waiting for access grant by the BS. In order to correctly detect the information corresponding to different users, the BS should uniquely identify the active users by checking activity factor $P$ before data detection can take place.

The following function is calculated for signaling overheads that is determined as [28], [36] in Eq. (35) as:

$$
\operatorname{sig}_{\mathrm{oh}} \triangleq C_{t} \times\left(N_{m t}+P N_{M N}\right)
$$

where $C_{t}$ is the time consumed between the transmission of the signal and its acknowledgment. $N_{m t}$ is the number of MNs which have movement to the target node, and $P$ is the activity factor denoted as the probability of $\mathrm{MN}$ being tracked that is chosen to be $1 . N_{M N}$ is the number of MNs currently handled by the target node.

Finally, we refer to the average rate of successful message delivery over a communication channel as the throughput. Such metric, denoted with $T_{a}$, is calculated as:

$$
T_{\mathrm{a}} \triangleq W \times \mathcal{R} \times\left(1-D_{H O \text { execution }}\right)
$$

where $\mathcal{R}$ is the achievable rate per unit bandwidth (in sec/Hz), $W$ denotes the system bandwidth of the channel and $D_{H O}$ execution is the the normalized average time wasted during the handover execution. It is the fraction of time where no data is transmitted to the test user. In order to calculate the throughput, we should eliminate the handover execution period. 
Table IV

LIST OF SIMULATION PARAMETERS.

\begin{tabular}{lll}
\hline Description & Parameter & Value \\
\hline System Bandwidth & $\mathrm{W}$ & $10-12 \mathrm{MHz}$ \\
UAV transmit power & $\mathrm{P}$ & $5 \mathrm{~W}$ \\
Exponent Path loss for UAV-user link & $\alpha_{\mathrm{UAV}}$ & 4 \\
Simulation area & $-\mathrm{MBS}$ & $10 \mathrm{~km}^{2}$ \\
Number of macro cell base station & $\mathrm{Aps}$ & 100 \\
Number of access points configured around each cell & $\mathrm{R}$ & 20 \\
Radio range of UAVs & $N_{0}$ & $500-800 \mathrm{~m}$ \\
Noise power spectral density & $h$ & $174 \mathrm{dBm} / \mathrm{Hz}$ \\
UAV altitude & $C_{t}$ & $200-550 \mathrm{ft}$ \\
Transmission constant & $C_{r}$ & $-11 \mathrm{Db}$ \\
Call request & $\mathrm{SINR}$ Threshold & $100 \mathrm{per}$ zone \\
Probabilistic SINR Threshold & $\mathrm{MU}$ & $-6 \mathrm{~dB}$ \\
Maximum number of active users & $\mathrm{Th}$ & 500 \\
Receiving Threshold & - & $1.17557 \mathrm{e}-10 \mathrm{~W}$ \\
Radio Propagation Model & - & Friis model \\
Antenna Type & - & Omni Antenna \\
Maximum Data Rate (WiMAX) & - & $1882 \mathrm{Kbps}$ \\
PHY Mode & - & $256 \mathrm{OFDM}$ \\
Maximum Data Rate (UMTS) & & $384 \mathrm{Kbps}$ \\
\hline
\end{tabular}

\section{Numerical Results and Discussion}

For performance comparison, four models namely SDNUAV-AP model, SDN random UAV model, the SDN enhanced UAV-based wireless network architecture [36] and proposed model without AP are used for comparison with the proposed model. In [36] model, a UAV module, which decided to shift traffic according to the network load and the active users' number, was invoked only when needed to reduce the burden of the controller. The SDN random UAV model, address the trade-off between the handover performance without selecting the best UAVs. There is a random UAV selection in handover process.

The reported results, shown in Fig. 7, prove that our architecture achieves significantly-lower latency than standard SDN-UAV network architecture, namely $-43.5 \%$.

Our architecture using $\mathrm{MIH}$ has a lower sensing delay to find the existing available networks. In our SDN-MIH-based UAV architecture, the SDN provided handover facilities in the UAVs as on-demand forwarding switches that supported wireless networks with lower handover latency.

Clearly, velocity has an essential influence on the handover delay. Indeed, in a wireless heterogeneous environment, rapid fluctuations of the received signal level may occur due to distortion or short-term shadowing of mobiles moving at high velocities.

Accordingly, Fig. 8 shows the average handover delay is $\approx 3.4 \mathrm{~ms}$ for a speed of $120 \mathrm{~km} / \mathrm{h}$ which is acceptable for a wireless network providing real-time services. Indeed, for instance, the IEEE considers $50 \mathrm{~ms}$ [50] as a tolerable maximum delay.

The high delay affects the entire cellular system and this can be lead to a decline in the QoS parameters to the end-users.

The processing delay has not considerable influence over the network performance of all the compared methodologies.

However, the main difference is caused by transmission and propagation delays.

Since our method is established on the MIH [35], it is comparatively fast and needs a lower time to get the necessary information about available networks. Also, in the designed architecture the number of UAVs are mapped in a single iteration. Therefore, the overall delays would reduce during the connectivity time with variation in the number of MNs.

Then, in Fig. 9 we investigate the capability of our framework in ensuring robustness with respect to different altitudes values $h$ (i.e. $250 \mathrm{ft}$ and $600 \mathrm{ft}$ in the considered experiments), namely to provide lower delays for a wide range of $h$ with respect to compared schemes.

As we can see, the altitude has an essential impact on network delays.

Indeed, the throughput coverage increases at low height and can thus increase the packets received at the users. Therefore, it can be mentioned that the proposed framework is able to offer enhanced services to users by means of a lower network (total) delay.

Indeed, SDN-MIH based UAV networks have better control and monitoring for network components, especially in ondemand forwarding switches.

The signaling overheads results, shown in Fig. 10, prove that our framework built on SDN-MIH has less signaling overhead with respect to the four baselines considered. For instance, in a setup with $1000 \mathrm{MNs}$, our approach achieves $-11 \%,-28.5 \%,-77.1 \%$ and $-77.1 \%$ signaling overheads than Sharma [36], SDN-UAV-AP, SDN-only-UAV, and SDNrandom-UAV, respectively.

Therefore, the numerical outcomes display that our proposed handover approach based on SDN-MIH-UAV outperforms other SDN-UAVs handover baselines.

The proposed model is optimized to select the UAVs that provide high throughput and a low packet-loss ratio.

Firstly, a rapid period is needed to trigger SDN-MIH-UAV, after that, the rate of packet delivery in SDN-MIH-UAV is improved over simulation time, even though when the SDNMIH-UAV decision solution has employed the throughput experienced by the mobile node is continuous without any interruption. This is because the SDN-MIH-UAV utilizes the optimized network selection that can select the best UAV in 


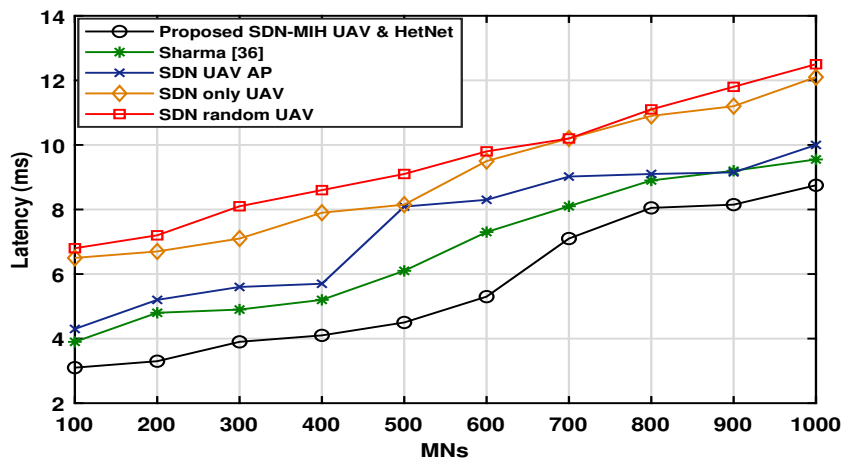

Figure 7. Handover latency (lat $\mathrm{HO}_{\mathrm{O}}$ ) vs. number of MNs.

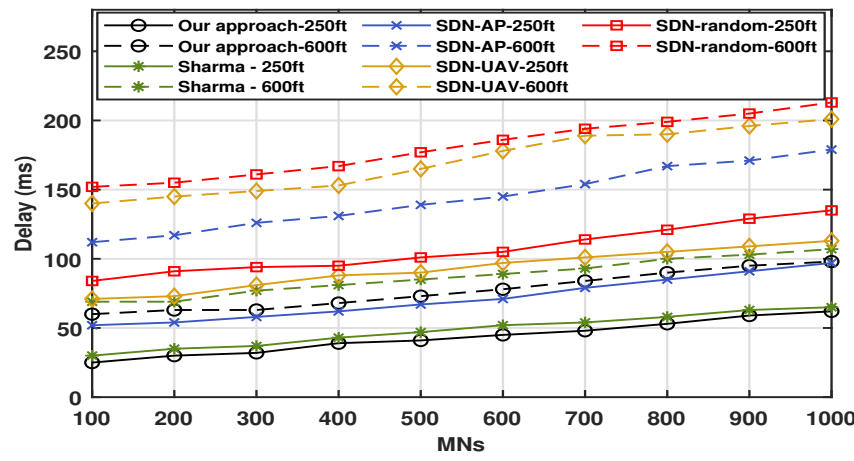

Figure 9. Total delay $\left(D_{\text {total }}\right)$ vs. number of MNs with different altitude values $(250$ and $600 \mathrm{ft})$.

a lower level of loss of packets and low delay in handover, thus growing the throughput. The loss of packets can affect throughput indirectly which shows the better throughput coverage with an increase in the users 'requirements in the specific macrocell.

Other methods suffer from high packet loss and as such offer low expansions in throughput mostly because of inappropriate handovers.

Delays in handovers also have negative effects on the throughput. Hence, since the SDN-MIH-UAV uses MIH protocol for improving QoS and for managing connectivity objects, there is also high throughput in the mobile node. Fig. 11 denotes the comparison in throughput experienced by the mobile node with respect to the increase in the UAVs the SDNMIH-UAV and other approaches. However, the existing SDNUAV method is depended on the exact identification of the demand areas only. So, it cannot guarantee higher throughput coverage generally. Actually, the altitude has significant effect on the coverage. Whenever the UAV decreases its height from a demand area, the distance decreases, and this matter will lead to throughput coverage increase.

Similarly, the number of failed handovers is examined versus the simulation time.

The total number of handovers performed and the number of failed handovers are also recorded.

The message "handover failure" is generated when target network sends a "handover failure" message as an unsuccessful response for the handover request message.

For HetNet mobility performance evaluations, the Radio

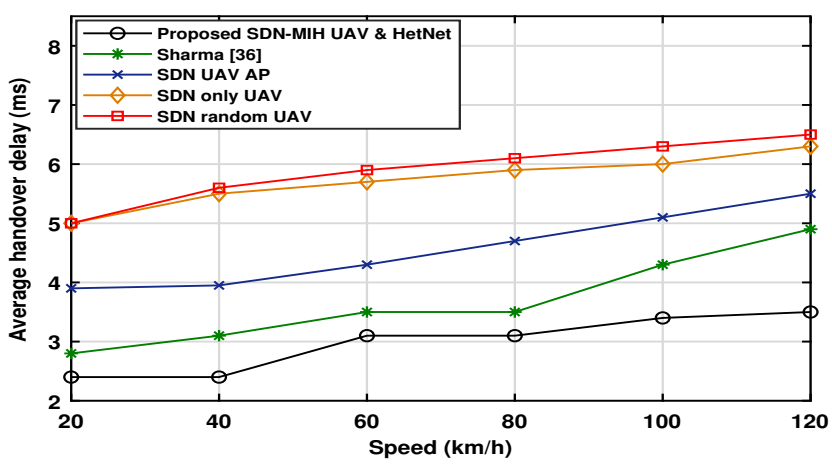

Figure 8. Average handover delay $\left(D_{h o}\right)$ vs. speed.

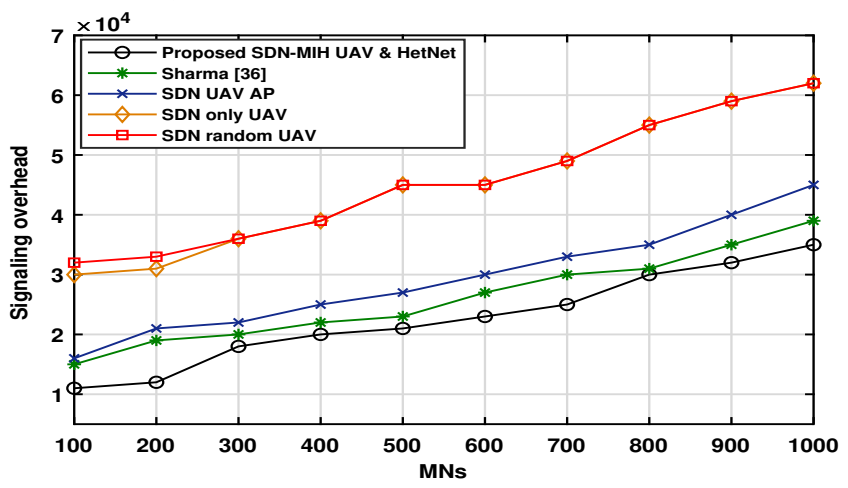

Figure 10. Signaling overheads ( $\mathrm{sig}_{\mathrm{oh}}$ ) vs. number of MNs.

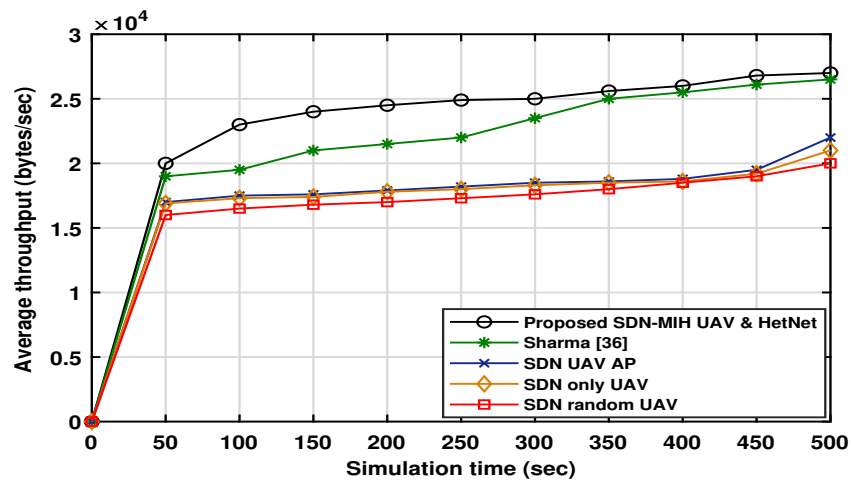

Figure 11. Average throughput $\left(T_{a}\right)$ vs. considered simulation time.

Link Failure (RLF) criterion and procedures are used to determine and control the handover failure. RLF handover provides a recovery mechanism when the handover signalling with the source cell fails due to poor radio conditions.

A handover is considered failed when the user unsuccessfully connects to the network because of poor radio conditions (e.g. experience low SINR). The user will then try to handback to its serving network. If this procedure also fails (the user has already moved out of the coverage area of serving network), then the user will be dropped. To measure the failed HOs, a dynamic timer is used to detect failures during the simulation run.

The timer is actualized each time that an event is produced 


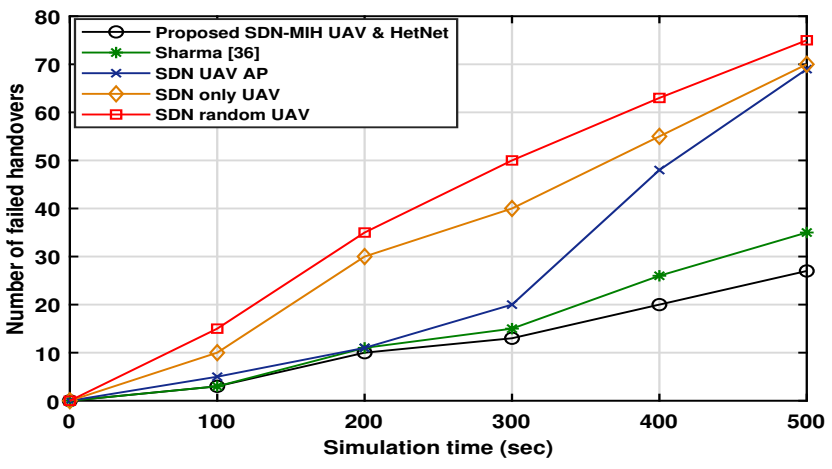

Figure 12. Cumulative number of $\mathrm{HO}$ failures vs. considered simulation time.

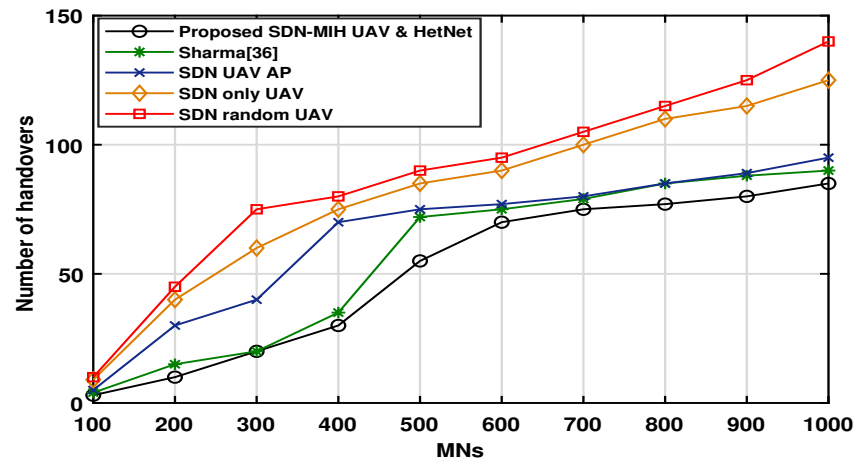

Figure 13. Number of handovers vs. number of MNs.

and then compares the current time with the time needed for decision making which is fixed at $0.05 \mathrm{~s}$ [36].

In contrast with other network selection methods, our approach can ensure a minimal number of failed HOs during this process. This is due to its network discovery mechanism based on the dynamic information granted by MIH. To this end, Fig. 12 presents the comparison of the proposed method and other methods focusing on failed HOs.

Interestingly, the total number of failed HOs of our approach is $\approx 10$ when considering the first 200 seconds of the simulation time. Conversely, the number of failed HOs incurred by other approaches is higher due to their less sophisticated triggering mechanisms (i.e. only RSS-based). Additionally, other approaches often initiate HOs which need quick interface switching across various systems.

Finally, as shown in Fig. 13, differently from other methods, our model incurs a lower number of HOs for a large range of MNs.

The main reasons for this effect are that proposed model adopts the MIH protocol, can provide higher data rates due to effective UAV capitalization, and leverages the AHP and the cost functions included in our handover decision model. Indeed, one of the reasons that can be desumed from the considered simulation observation is the inappropriate handover triggering caused by the RSS by the compared methods.

In summary, the aforementioned results have shown the appeal of our designed framework (SDN-MIH UAVs \&(HetNets)) over the four considered baselines in terms of both metrics directly related to the handover process (i.e. lat $\mathrm{HO}_{\mathrm{O}}$, $D_{\text {total }}$ and sigoh $_{\text {oh }}$ and to the overall communication system (i.e. $T_{a}$ ). The analysis has taken into account a large span of MNs numerosity scenarios and UAV types (associated to different speed values).

\section{Conclusions And Future Directions}

In this study, we focused on providing seamless mobility over UAV-assisted heterogeneous networks and proposed a hybrid model for managing and optimizing handover in each iteration among UAVs. We used game theory method to design competitive or cooperative conditions among decision makers in a heterogeneous environment. Hence, a model based on game theory was designed for network selection mechanism during vertical handover process between UAVs. The proposed model can organize efficient decision making in handover process by decreasing the end-to-end delay, handover latency, and signaling overheads. Additionally, we proposed an efficient scheme, based on SDN-MIH network, for handover management in heterogeneous wireless networks. In our framework, the standard SDN architecture was developed which uses the control layer for managing the UAVs as the forwarding switch in the data layer and the UAV networks were powered by MIH to transfer real-time data feeds in conjunction with other networks, such as WiFi, WiMAX and LTE. The proposed novel scheme of SDN-MIH-UAVs is able to improve network selection for vertical handovers by taking into account user preferences and mobile QoS requirements. The key problem for efficient mobility management is the unavailability of accurate information of the available networks in an uncertain environment. If the mobile node knew a-priori which network offers the best long-term performance, it would choose that network. This issue can avoid frequent handovers which lead to energy efficiency while achieving optimal energy consumption for service. To address this challenge, in future we plan to design UAV-based energy efficient mobility management scheme by reinforcement learning techniques enabling drones to service their ground users and improve the handover performance in terms of energy usage, call blocking probability (CBP), handover failure probability, and average waiting time in the queue. In addition, we will analytically study the calculation of handover failure probabilities of the proposed algorithm.

\section{ACKNOWLEDGMENT}

The authors wish to thank the Ministry of Higher Education Malaysia and Universiti Kebangsaan Malaysia (grant ID: GGPM-2020-029 and grant ID: PP-FTSM-2020) for supporting and funding this work.

\section{REFERENCES}

[1] T. M. Duong and S. Kwon, "Vertical handover analysis for randomly deployed small cells in heterogeneous networks," IEEE Transactions on Wireless Communications, vol. 19, no. 4, pp. 2282-2292, 2020.

[2] S. Li, D. Grace, Y. Liu, J. Wei, and D. Ma, "Overlap area assisted call admission control scheme for communications system," IEEE Transactions on Aerospace and Electronic Systems, vol. 47, no. 4, pp. 2911-2920, 2011. 
[3] K.-N. Park, J.-H. Kang, B.-M. Cho, K.-J. Park, and H. Kim, "Handover management of net-drones for future internet platform," International Journal of Distributed Sensor Networks, vol. 12, no. 3, p. 5760245, 2016.

[4] Q. Huang, Y.-C. Huang, K.-T. Ko, and V. B. Iversen, "Loss performance modeling for hierarchical heterogeneous wireless networks with speed-sensitive call admission control," IEEE Transactions on Vehicular Technology, vol. 60, no. 5, pp. 2209-2223, 2011.

[5] P. Zhan, K. Yu, and A. L. Swindlehurst, "Wireless relay communications with unmanned aerial vehicles: Performance and optimization," IEEE Transactions on Aerospace and Electronic Systems, vol. 47, no. 3, pp. 2068-2085, 2011.

[6] M. Mozaffari, W. Saad, M. Bennis, and M. Debbah, "Efficient deployment of multiple unmanned aerial vehicles for optimal wireless coverage," IEEE Communications Letters, vol. 20, no. 8, pp. 1647-1650, 2016.

[7] J. A. Mahal, A. Khawar, A. Abdelhadi, and T. C. Clancy, "Spectral coexistence of MIMO radar and MIMO cellular system," IEEE Transactions on Aerospace and Electronic Systems, vol. 53, no. 2, pp. 655-668, 2017.

[8] O. K. Sahingoz, "Networking models in flying ad-hoc networks (FANETs): Concepts and challenges," Springer Journal of Intelligent \& Robotic Systems, vol. 74, no. 1-2, pp. 513-527, 2014.

[9] I. D. Moscholios, V. G. Vassilakis, M. D. Logothetis, and A. C. Boucouvalas, "State-dependent bandwidth sharing policies for wireless multirate loss networks," IEEE Transactions on Wireless Communications, vol. 16, no. 8, pp. 5481-5497, 2017.

[10] R. W. Beard, D. Kingston, M. Quigley, D. Snyder, R. Christiansen, W. Johnson, T. McLain, and M. Goodrich, "Autonomous vehicle technologies for small fixed-wing UAVs," Journal of Aerospace Computing, Information, and Communication, vol. 2, no. 1, pp. 92-108, 2005.

[11] S. Koulali, E. Sabir, T. Taleb, and M. Azizi, "A green strategic activity scheduling for UAV networks: A sub-modular game perspective," IEEE Communications Magazine, vol. 54, no. 5, pp. 58-64, 2016

[12] A. E. E. A. Abdulla, Z. M. Fadlullah, H. Nishiyama, N. Kato, F. Ono, and R. Miura, "Toward fair maximization of energy efficiency in multiple UAS-aided networks: A game-theoretic methodology," IEEE Transactions on Wireless Communications, vol. 14, no. 1, pp. 305-316, 2015.

[13] Z. Han, D. Niyato, W. Saad, T. Başar, and A. Hjørungnes, Game theory in wireless and communication networks: theory, models, and applications. Cambridge University press, 2012.

[14] A. Al-Hourani, S. Kandeepan, and S. Lardner, "Optimal LAP altitude for maximum coverage," IEEE Wireless Communications Letters, vol. 3, no. 6, pp. 569-572, 2014

[15] H. Jiang, Z. Zhang, L. Wu, and J. Dang, "Three-dimensional geometrybased UAV-MIMO channel modeling for a2g communication environments," IEEE Communications Letters, vol. 22, no. 7, pp. 1438-1441, 2018.

[16] H. Jiang, Z. Zhang, and G. Gui, "Three-dimensional non-stationary wideband geometry-based UAV channel model for A2G communication environments," IEEE Access, vol. 7, pp. 26 116-26122, 2019.

[17] G. A. Akpakwu, B. J. Silva, G. P. Hancke, and A. M. Abu-Mahfouz, "A survey on $5 \mathrm{G}$ networks for the Internet of Things: Communication technologies and challenges," IEEE Access, vol. 6, pp. 3619-3647, 2017.

[18] D.-I. Curiac, "Towards wireless sensor, actuator and robot networks: Conceptual framework, challenges and perspectives," Elsevier Journal of Network and Computer Applications, vol. 63, pp. 14-23, 2016.

[19] H.-H. Shuai, D.-N. Yang, W.-H. Cheng, and M.-S. Chen, "MobiUP: An upsampling-based system architecture for high-quality video streaming on mobile devices," IEEE Transactions on Multimedia, vol. 13, no. 5, pp. 1077-1091, 2011

[20] Y. Zeng, R. Zhang, and T. J. Lim, "Wireless communications with unmanned aerial vehicles: Opportunities and challenges," IEEE Communications Magazine, vol. 54, no. 5, pp. 36-42, 2016

[21] M. Sara, I. Jawhar, and M. Nader, "A softwarization architecture for UAVs and WSNs as part of the cloud environment," in IEEE International Conference on Cloud Engineering Workshop (IC2EW), 2016, pp. $13-18$.

[22] A. Merwaday and I. Guvenc, "UAV assisted heterogeneous networks for public safety communications," in IEEE wireless communications and networking conference workshops (WCNCW), 2015, pp. 329-334.

[23] Z. M. Fadlullah, D. Takaishi, H. Nishiyama, N. Kato, and R. Miura, "A dynamic trajectory control algorithm for improving the communication throughput and delay in UAV-aided networks," IEEE Network, vol. 30 no. 1 , pp. 100-105, 2016.

[24] N. McKeown, T. Anderson, H. Balakrishnan, G. Parulkar, L. Peterson, J. Rexford, S. Shenker, and J. Turner, "Openflow: enabling innovation in campus networks," ACM SIGCOMM Computer Communication Review, vol. 38 , no. 2, pp. 69-74, 2008.

[25] H. Kim and N. Feamster, "Improving network management with software defined networking," IEEE Communications Magazine, vol. 51, no. 2, pp. 114-119, 2013.

[26] B. Yang, Y. Wu, X. Chu, and G. Song, "Seamless handover in softwaredefined satellite networking," IEEE Communications Letters, vol. 20, no. 9, pp. 1768-1771, 2016.

[27] H. Iqbal, J. Ma, K. Stranc, K. Palmer, and P. Benbenek, "A softwaredefined networking architecture for aerial network optimization," in IEEE NetSoft Conference and Workshops (NetSoft), 2016, pp. 151-155.

[28] V. Sharma, M. Bennis, and R. Kumar, "UAV-assisted heterogeneous networks for capacity enhancement," IEEE Communications Letters, vol. 20, no. 6, pp. 1207-1210, 2016

[29] Z. Han, A. L. Swindlehurst, and K. R. Liu, "Optimization of MANET connectivity via smart deployment/movement of unmanned air vehicles," IEEE Transactions on Vehicular Technology, vol. 58, no. 7, pp. 35333546, 2009.

[30] V. Sharma, K. Srinivasan, H.-C. Chao, K.-L. Hua, and W.-H. Cheng, "Intelligent deployment of UAVs in 5G heterogeneous communication environment for improved coverage," Elsevier Journal of Network and Computer Applications, vol. 85, pp. 94-105, 2017.

[31] M. Mozaffari, W. Saad, M. Bennis, Y.-H. Nam, and M. Debbah, "A tutorial on UAVs for wireless networks: Applications, challenges, and open problems," IEEE Communications Surveys \& Tutorials, 2019.

[32] M. E. Mkiramweni, C. Yang, J. Li, and W. Zhang, "A survey of game theory in unmanned aerial vehicles communications," IEEE Communications Surveys \& Tutorials, vol. 21, no. 4, pp. 3386-3416, 2019.

[33] V. Radonjić and V. Aćimović-Raspopović, "Responsive pricing modeled with Stackelberg game for next-generation networks," Springer Annals of Telecommunications-Annales des Télécommunications, vol. 65, no. 7-8, pp. 461-476, 2010.

[34] U. Challita, W. Saad, and C. Bettstetter, "Interference management for cellular-connected UAVs: A deep reinforcement learning approach," IEEE Transactions on Wireless Communications, vol. 18, no. 4, pp. 2125-2140, 2019.

[35] V. Sharma, J. Guan, J. Kim, S. Kwon, I. You, F. Palmieri, and M. Collotta, "MIH-SPFP: MIH-based secure cross-layer handover protocol for fast proxy mobile IPv6-IoT networks," Journal of Network and Computer Applications, vol. 125, pp. 67-81, 2019.

[36] V. Sharma, F. Song, I. You, and H.-C. Chao, "Efficient management and fast handovers in software defined wireless networks using UAVs," IEEE Network, vol. 31, no. 6, pp. 78-85, 2017.

[37] E. T. Michailidis, N. Nomikos, P. S. Bithas, D. Vouyioukas, and A. G. Kanatas, "Optimal 3-d aerial relay placement for multi-user mimo communications," IEEE Transactions on Aerospace and Electronic Systems, vol. 55, no. 6, pp. 3218-3229, 2019.

[38] M. Ohleger, G. G. Xie, and J. H. Gibson, "Extending UAV video dissemination via seamless handover: A proof of concept evaluation of the IEEE 802.21 standard," in 46th IEEE Hawaii International Conference on System Sciences (HICSS), 2013, pp. 5106-5114.

[39] T. Mahmoodi and S. Seetharaman, "On using a SDN-based control plane in 5G mobile networks," in Wireless World Research Forum, 32nd Meeting, 2014.

[40] S. Jeong, O. Simeone, and J. Kang, "Mobile edge computing via a UAVmounted cloudlet: Optimization of bit allocation and path planning," IEEE Transactions on Vehicular Technology, vol. 67, no. 3, pp. 20492063, 2018.

[41] J. Lyu, Y. Zeng, R. Zhang, and T. J. Lim, "Placement optimization of UAV-mounted mobile base stations," IEEE Communications Letters, vol. 21 , no. 3, pp. 604-607, 2017.

[42] K. P. Yoon and C.-L. Hwang, Multiple attribute decision making: an introduction. Sage publications, 1995, vol. 104.

[43] T. L. Saaty, "Decision-making with the AHP: Why is the principal eigenvector necessary," Elsevier European Journal of Operational Research, vol. 145 , no. 1, pp. 85-91, 2003.

[44] X. Cheng and Y. Li, "A 3-d geometry-based stochastic model for uav-mimo wideband nonstationary channels," IEEE Internet of Things Journal, vol. 6, no. 2, pp. 1654-1662, 2018.

[45] C. Shepard, A. Rahmati, C. Tossell, L. Zhong, and P. Kortum, "Livelab: measuring wireless networks and smartphone users in the field," $A C M$ SIGMETRICS Performance Evaluation Review, vol. 38, no. 3, pp. 1520, 2011.

[46] M. Mozaffari, W. Saad, M. Bennis, and M. Debbah, "Unmanned aerial vehicle with underlaid device-to-device communications: Performance and tradeoffs," IEEE Transactions on Wireless Communications, vol. 15, no. 6, pp. 3949-3963, 2016. 
[47] C. A. C. Cortez and J. C. Pinto, "Improvement of Karush-Kuhn-Tucker conditions under uncertainties using robust decision making indexes," Applied Mathematical Modelling, vol. 43, pp. 630-646, 2017.

[48] C. Guimaraes, D. Corujo, R. L. Aguiar, F. Silva, and P. Frosi, "Empowering software defined wireless networks through media independent handover management," in IEEE Global Communications Conference (GLOBECOM), 2013, pp. 2204-2209.

[49] M. Rezaee and M. H. Y. Moghaddam, "SDN-based quality of service networking for wide area measurement system," IEEE Transactions on Industrial Informatics, 2019.

[50] T. Bilen, B. Canberk, and K. R. Chowdhury, "Handover management in software-defined ultra-dense 5G networks," IEEE Network, vol. 31, no. 4, pp. 49-55, 2017.

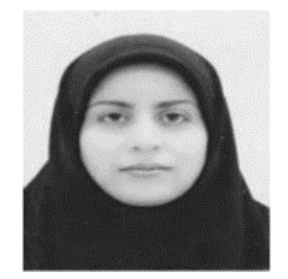

Shidrokh Goudarzi received her Ph.D. degree in communication system and wireless network from Malaysia-Japan International Institute of Technology (MJITT), Universiti Teknologi Malaysia (UTM). In 2014, She received three year full scholarship to study Ph.D. at (UTM).Then, She joined the Department of Advanced Informatics School at Universiti Teknologi Malaysia as a Postdoctoral Fellow from 2018 to 2019. Currently, she is a senior lecturer at Universiti Kebangsaan Malaysia (UKM). She also serves as reviewer for Canadian Journal of Electrical and Computer Engineering, KSII Transactions on Internet and Information Systems Journal,Journal of Engineering and Technological Sciences, Mathematical Problems in Engineering and IEEE Access. Her research interests are in wireless networks, artificial intelligence, machine learning, next generation networks, Internet of Things (Iot) and Mobile/distributed/Cloud Computing.

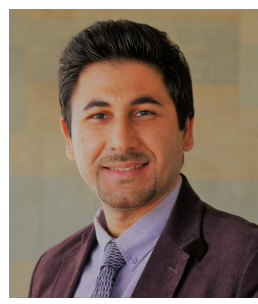

Mohammad Hossein Anisi (M'14-SM'19) is an Assistant Professor at the School of Computer Science and Electronic Engineering, University of Essex and head of Internet of Everything (IoE) Laboratory (essex-ioe.com). Prior to that, he worked as a Senior Research Associate at University of East Anglia, UK and Senior Lecturer at University of Malaya, Malaysia where he received "Excellent Service Award" for his achievements. His research has focused specifically on real world application domains such as energy management, transportation, healthcare and other potential life domains. As a computer scientist, he has designed and developed novel architectures and routing protocols for Internet of Things (IoT) enabling technologies including wireless sensor and actuator networks, vehicular networks, heterogeneous networks, body area networks and his research results have directly contributed to the technology industry. He has strong collaboration with industry and working with several companies in the UK with the focus on monitoring and automation systems based on IoT concept capable of reliable and seamless generation, transmission, processing and demonstration of data.He has published more than 80 articles in high quality journals and several conference papers and won two medals for his innovations from PECIPTA 2015 and IIDEX 2016 expositions. He has received several International and national funding awards for his fundamental and practical research as PI and Co-I. Dr Anisi is an associate editor of a number of journals including "IEEE Access", "Ad Hoc \& Sensor Wireless Networks", "IET Wireless Sensor Systems", "International Journal of Distributed Sensor Networks", "KSII Transactions on Internet and Information Systems journals and "Journal of Sensor and Actuator Networks". He has been guest editor of special issues of the journals and Lead organizer of special sessions and workshops at IEEE conferences such as IEEE CAMAD, IEEE PIMRC, IEEE VTC and etc. He has been also serving as executive/technical committee member of several conferences. Hossein is Fellow of Higher Education Academy and Senior Member of IEEE. He is also a technical committee member of Finnish-Russian University Cooperation in Telecommunications (FRUCT), Senior Member of Institute of Research Engineers and Doctors (the IRED), Member of ACM, IEEE Council on RFID, IEEE Sensors Council, IEEE Systems Council and International Association of Engineers (IAENG).

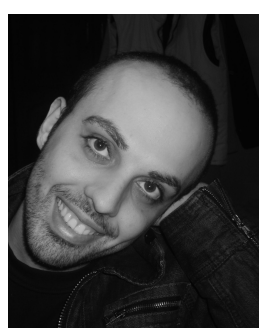

Domenico Ciuonzo (S'11-M'14-SM'16) is currently an Assistant Professor at University of Napoli Federico II, Italy. He holds a Ph.D. in Electronic Engineering from University of Campania "L. Vanvitelli", Italy and, from 2011, he has held several visiting researcher appointments. Since 2014 he is (senior) editor of several IEEE, IET and ELSEVIER journals. His research interests include data fusion, statistical signal processing, wireless sensor networks, Internet of Things, traffic analysis and machine learning.

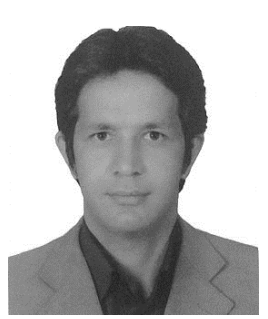

Seyed Ahmad Soleymani received his Ph.D. degree in computer science from faculty of engineering, Universiti Teknologi Malaysia (UTM). He received his M.S degree from the Department of Computer Engineering, Islamic Azad University, Iran and B.S. degree from the Department of Computer Engineering, Sadjad University, Iran. His research interests are in the area of Wireless Sensor Network (WSN), Mobile Ad Hoc Network (MANET), Vehicular Ad Hoc Network (VANET), Internet of Things and Nano Things (IoT and IoNT), Visible Light Communication (VLS), Intelligent Algorithms (IAs), Big Data and Machine Learning.

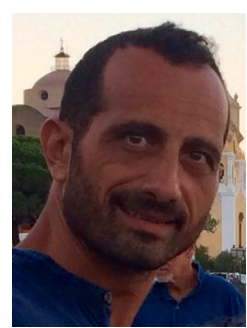

Antonio Pescapé (SM'09) is a Full Professor of computer engineering at the University of Napoli Federico II. His work focuses on Internet technologies and specifically on measurement, monitoring, and analysis of the Internet. He has co-authored more than 200 papers and is the recipient of a number of awards. He is involved in several research projects on Internet Technologies and he is reviewer and evaluator of research projects for international agencies, governments, and EU commission. 